\title{
Multiscale modelling and analysis of lithium-ion battery charge and discharge
}

\author{
G. Richardson - G. Denuault • C. P. Please
}

Received: 12 August 2009 / Accepted: 13 February 2011

(C) Springer Science+Business Media B.V. 2011

\begin{abstract}
A microscopic model of a lithium battery is developed, which accounts for lithium diffusion within particles, transfer of lithium from particles to the electrolyte and transport within the electrolyte assuming a dilute electrolyte and Butler-Volmer reaction kinetics. Exploiting the small size of the particles relative to the electrode dimensions, a homogenised model (in agreement with existing theories) is systematically derived and studied. Details of how the various averaged quantities relate to the underlying geometry and assumptions are given. The novel feature of the homogenisation process is that it allows the coefficients in the electrode-scale model to be derived in terms of the microscopic features of the electrode (e.g. particle size and shape) and can thus be used as a systematic way of investigating the effects of changes in particle design. Asymptotic methods are utilised to further simplify the model so that one-dimensional behaviour can be described with relatively simpler expressions. It is found that for low discharge currents, the battery acts almost uniformly while above a critical current, regions of the battery become depleted of lithium ions and have greatly reduced reaction rates leading to spatially nonuniform use of the electrode. The asymptotic approximations are valid for electrode materials where the OCV is a strong function of intercalated lithium concentration, such as $\mathrm{Li}_{x} \mathrm{C}_{6}$, but not for materials with a flat discharge curve, such as $\mathrm{LiFePO}_{4}$.
\end{abstract}

Keywords Butler-Volmer equation $\cdot$ Electrolyte $\cdot$ Homogenisation $\cdot$ Matched asymptotic expansions

\section{Introduction}

There is a considerable amount of interest shown in designing lithium-ion batteries with the aim of increasing their efficiency of storage and the speed at which they can be charged and discharged. In seeking to understand this behaviour, a number of theoretical approaches have been taken to simulate the operation of such batteries typically based on the seminal study by Newman [1, Chaps. 11-12, 22] (see, e.g. [2-11]). In this article, these ideas are further explored to derive analytical expressions for the discharge and charging characteristics.

G. Richardson $(\varangle) \cdot$ C. P. Please

School of Mathematics, University of Southampton, Southampton SO17 1BJ, UK

e-mail: G.Richardson@soton.ac.uk

G. Denuault

School of Chemistry, University of Southampton, Southampton SO17 1BJ, UK 


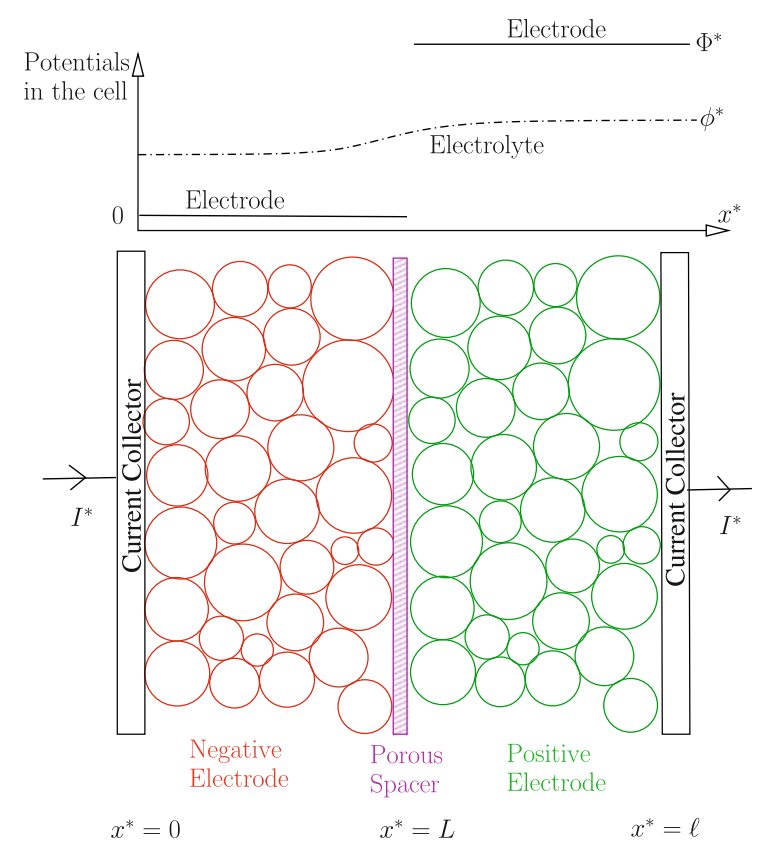

Fig. 1 A schematic of the configuration of the battery. The electrodes consist of particles packed together. These are immersed in the electrolyte, and the contact between the two electrodes is avoided by placing a porous spacer between them through which only the electrolyte can percolate. During discharge, the current flowing out of the positive electrode drives the extraction of Li cations from the negative electrode particles, the transport (by diffusion and migration) of these ions across the separator into the positive electrode and their subsequent insertion into the positive electrode particles. The transport of $\mathrm{Li}^{+}$is accompanied by the movement of electrons from the negative electrode particles to the negative electrode current collector and from the positive electrode current collector to the positive electrode particles. The upper panel is a schematic of the potentials in the battery: solid line shows the potential in the electrode particles while the dashed line shows that in the electrolyte

The lithium cell studied here has two electrodes, a negative one (the anode in discharge mode) and a positive one (the cathode in discharge mode), each composed of small particles of electrode material compressed together. The particles are presumed to be electrically connected via some conducting medium (e.g. graphite powder utilised to improve electrode conductivity) so that all the negative electrode (anode) particles connect to the negative electrode current collector and all the positive electrode (cathode) particles connect to the positive electrode current collector. However, the anode and cathode particles are kept separated by a thin electrically insulating porous separator. The entire battery is bathed in an electrolyte containing lithium ions (see Fig. 1). Lithium is either stored in metallic form or in solid solution (inside an insertion material) on the negative electrode and in solid solution on the positive electrode [12].

During discharge, a reaction occurs on the surface of the negative electrode particles that releases $\mathrm{Li}^{+}$ions from the material into the electrolyte while a corresponding reaction occurs on the surface of the positive electrode particles in which $\mathrm{Li}^{+}$are absorbed from the electrolyte. These reactions drive a flux of ions through the electrolyte, and the separator, from the negative to the positive electrode. They also result in the build up of positive charge on the positive electrode and negative charge on the negative electrode leading to a potential difference that can be utilised to drive an electric current. Typically, it is hypothesised that the reaction rate depends upon the $\mathrm{Li}$ concentration on the electrode surface, $\mathrm{Li}^{+}$concentration in the adjacent electrolyte and the potential drop between the electrode and electrolyte (see, e.g. $[8,13,14]$ ). The reaction, and thus the corresponding release of charge, may thus be limited by diffusion of $\mathrm{Li}^{+}$in the electrolyte, diffusion of $\mathrm{Li}$ in the electrode material or by the electrical resistivity of the electrolyte or electrodes, and it is important to investigate the relative importance of these processes. 
A large variety of materials have been developed for use as positive electrodes in lithium batteries since the 1970s (see [15]). Currently, commercially available Li-batteries use $\mathrm{LiCoO}_{2}$. However, the rarity of cobalt makes this relatively expensive and limits its use to small cells. Recent development has focussed on materials for use in large high-power applications (such as electric vehicles) [16] with much of the present focus being on $\mathrm{LiFePO}_{4}$ [17]. This material is characterised by a very flat discharge-voltage curve with practically no drop in voltage until the material is almost entirely discharged. This observation has led to the hypothesis that the transition between lithium-rich and lithium-poor states, in $\mathrm{LiFePO}_{4}$, is characterised by a phase transition as $\mathrm{Li}$ (de-)intercalates [17]. In addition, this material has a highly anisotropic crystal structures in which the diffusion of lithium takes place almost entirely parallel to the $b$-axis of the crystal [18]. The exact details of $\mathrm{Li}$ transport within a $\mathrm{LiFePO}_{4}$ cathode are still contested and a variety of different models have been proposed to describe this process (e.g. [17,19]). Standard single phase diffusion models have been utilised to describe Li transport in a variety of other materials (see, e.g. $[2,4,5,7,8]$ ), and this is the approach that we will adopt here.

The standard Li-ion battery negative electrode material is graphite. This material has the advantages of being highly conducting and of not exhibiting particularly large strains as Li (de-)intercalates but suffers from a relatively low energy density [20]. Higher energy density materials are available (e.g. Al or Si), and being developed [21], but they exhibit very large strains on (de-)intercalation of lithium which can result in eventual failure of the material due to cracking.

There is currently a considerable impetus to develop electrodes with a carefully engineered nanostructure with the aim of enhancing global battery performance [21-23], typically for applications requiring high (dis)charge rates. This typically enforces the use of a narrow cathode. Better theoretical understanding of this regime gives the prospect of designing non-uniform microstructures that are better able to cope with high current discharges.

A variety of macroscopic models have been developed to describe the behaviour of batteries with particulate electrodes. In the case of Li-ion batteries, these include the study of John Newman and his co-workers [4,5,7,8] and Botte et al. [2] while other systems have been treated in a similar manner, for example, by Chen and Cheh [24]. All these approaches replace the effect of the microstructure by a few phenomenological parameters. Given the current interest in designing the microstructure of the battery, a modelling approach that is capable of directly relating the geometry of the microstructure to the coefficients in a macroscopic model of battery behaviour would provide a valuable tool with which to design microstructures for particular purposes.

A microscopic mathematical model of the electrochemical processes occurring inside the battery, on the particle lengthscale, will be presented. The model consists of five distinct elements. These comprise (i) the diffusion of lithium in a positive electrode particle, (ii) the transfer of lithium between the positive electrode particles and the electrolyte, (iii) the transport of lithium cations through the electrolyte, (iv) the transfer of lithium from the electrolyte to the negative electrode particles, and (v) the diffusion of lithium within negative electrode particles. A homogenisation technique is then applied to the microscopic model to derive a simpler homogeneous model describing the averaged electrochemical behaviour of the battery over the cell lengthscale. The novel feature of the homogenisation process is that it allows the coefficients in the electrode-scale model to be derived in terms of the microscopic features of the electrode (e.g. particle size and shape) and can thus be used as a systematic way of investigating the effects of changes in particle design. We shall exploit this feature to investigate the effects of varying particle geometries in a subsequent article. By focussing on the physically realistic case in which diffusion times across the electrode particles are quick in comparison to those across the electrolyte and where the open circuit voltage (OCV) is much greater than the thermal voltage (about $0.025 \mathrm{~V}$ ), it can be shown that the voltage drop across the cell is close to the OCV except at extremely high discharge/charge rates. At low discharge rates, lithium ions are inserted into the positive electrode particles uniformly throughout the electrode (conversely at low charge rates, they are inserted uniformly throughout the negative electrode). However, at moderately high discharge rates, diffusion through the electrolyte is insufficient to ensure spatially uniform deposition of lithium ions in the positive electrode particles, with those farthest from the separator receiving virtually no lithium ions (conversely at moderately high charge rates, spatial deposition of lithium ions in the negative electrode is nonuniform). Since each electrode particle has a given capacity to absorb 
lithium, this results in a decreased fraction of utilised stored energy that can be achieved by a battery operating at high currents. In this context, we note the recent study of Johns et al. [25], which treats this aspect of lithium ion battery operation in which a simple model is used, in conjunction with experiments, to illustrate the effects of salt starvation at high rates of discharge. For comparison with the results of [25], we have included plots of the fraction of the utilised stored energy (as a function of the discharge current) calculated based on our model.

In Sect. 2, we formulate a microscopic model describing the transfer of lithium from an electrode particle into the electrolyte and its subsequent motion. We investigate this model in the scenario where large aggregations of small electrode particles are utilised to form the electrodes in a battery in Sect. 3. In particular, we show that, where the diffusion of the lithium within the particles is fast, the microscopic model may be systematically approximated by a much simpler macroscopic model operating over the scale of the whole battery. In Sect. 4, we look at further simplifications to this macroscopic model based on the likely parameter regimes for a real device. Finally, in Sect. 5, we draw our conclusions.

\section{Mathematical model}

We consider an electrolyte containing lithium ions (the cation) and some negative ion species (the anion), with valency 1 , and with concentrations $c_{\mathrm{p}}^{*}$ and $c_{\mathrm{n}}^{*}$, respectively. In this article, a $*$ appended to a variable denotes that it is dimensional. At the surface of the particles in both electrodes, reactions take place which transport lithium from an ionic state in the electrolyte to an intercalated state in the insertion material within the electrode particles, which can be summarised in the form

$\operatorname{Im}+\mathrm{Li}^{+}+e^{-} \leftrightharpoons \mathrm{ImLi}$

where Im and ImLi denote, respectively, the insertion material before and after $\mathrm{Li}$ insertion.

The Geometry. The geometry of the problem is illustrated in Fig. 1. In this context, the negative electrode particles occupy the region $0<x^{*}<L$; there is then a thin spacer on the other side of which, in $L<x^{*}<\ell$; and there is a region occupied by the positive electrode particles. Both negative electrode and positive electrode particles are surrounded by electrolyte which can freely permeate the porous spacer. We denote the region occupied by the negative electrode particles by $\Omega_{\mathrm{a}}$, that occupied by positive electrode particles by $\Omega_{\mathrm{c}}$ and that by the electrolyte by $\Omega_{\mathrm{e}}$. This is illustrated in Fig. 2. Furthermore, we take the normal vector $\boldsymbol{n}$ to the interface $\partial \Omega_{\mathrm{ae}}$ between the negative electrode particles, and the electrolyte and the interface $\partial \Omega_{\mathrm{ce}}$ between the positive electrode particles and the electrolyte to be directed towards the electrolyte (see Fig. 2).

Ion transport in a dilute electrolytic solution around particles. We consider the electrolyte to be adequately described by a mathematical model of a dilute binary electrolyte. In all but extremely narrow Debye layers (typical width $1 \mathrm{~nm}$ ) about the surface of the electrodes, charge neutrality is almost exactly satisfied-that is $c_{\mathrm{p}}^{*} \approx c_{\mathrm{n}}^{*}$ (see, e.g. [26] for further details). This motivates us to write $c_{\mathrm{p}}^{*} \approx c^{*}$ and $c_{\mathrm{n}}^{*} \approx c^{*}$ and write down the following (approximate) conservation equations, in the standard fashion, for the two ion species in the bulk of the electrolyte away from the Debye layers:
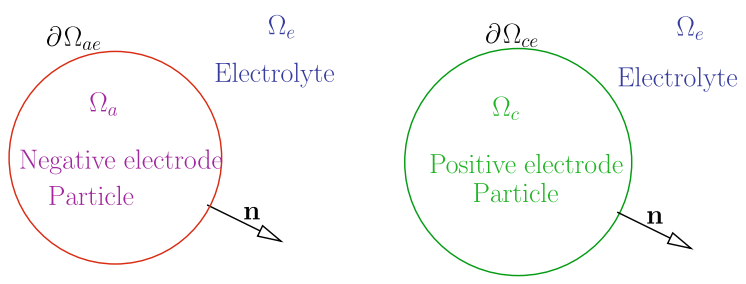

Fig. 2 Schematic representation of the mathematical domains corresponding to the electrolyte $\Omega_{\mathrm{e}}$, the negative and positive electrode particles, $\Omega_{\mathrm{a}}$ and $\Omega_{\mathrm{c}}$ respectively, and the interfaces with the surrounding electrolyte, $\partial \Omega_{\mathrm{ae}}$ and $\partial \Omega_{\mathrm{ce}}$ 
$\left.\begin{array}{lc}\frac{\partial c^{*}}{\partial t^{*}}+\nabla^{*} \cdot \boldsymbol{q}_{\mathrm{p}}^{*}=0, & \boldsymbol{q}_{\mathrm{p}}^{*}=-D_{\mathrm{p}}\left(\nabla^{*} c^{*}+\frac{F}{R_{\mathrm{g}} T} c^{*} \nabla^{*} \phi^{*}\right) \\ \frac{\partial c^{*}}{\partial t^{*}}+\nabla^{*} \cdot \boldsymbol{q}_{\mathrm{n}}^{*}=0, & \boldsymbol{q}_{\mathrm{n}}^{*}=-D_{\mathrm{n}}\left(\nabla^{*} c^{*}-\frac{F}{R_{\mathrm{g}} T} c^{*} \nabla^{*} \phi^{*}\right)\end{array}\right\} \quad$ in $\Omega_{\mathrm{e}}$.

Here, the left-hand side equations represent the conservation of the two ion species with respective ion fluxes $\left(\boldsymbol{q}_{\mathrm{p}}^{*}\right.$ and $\boldsymbol{q}_{\mathrm{n}}^{*}$ ) and these fluxes are defined by the right-hand side equations. In these fluxes, the first term represents a diffusive flux of ions, while the second represents an advective flux created by the electric field, $\boldsymbol{E}^{*}=-\nabla^{*} \phi^{*}$, acting on the ions, where $\phi^{*}$ is the electric potential in the electrolyte. The parameters $D_{\mathrm{p}}, D_{\mathrm{n}}, F, R_{\mathrm{g}}$ and $T$ are the diffusion coefficients of the positive and negative ions, Faraday's constant, the universal gas constant and absolute temperature, respectively. More details of the physical significance of these equations can be found, for example, in [26]. It is also useful to write down an equation for the current density $j^{*}$ in terms of the ion fluxes

$\boldsymbol{j}^{*}=F\left(\boldsymbol{q}_{\mathrm{p}}^{*}-\boldsymbol{q}_{\mathrm{n}}^{*}\right)$.

The electrodes. Within the electrode particles lithium is in an uncharged mode and is transported by diffusion becuase of concentration gradients. At the surface of the particles, the lithium is converted to ionic form as it is released into the electrolyte by the surface electrode reaction (alternatively this process takes place in reverse). We denote the lithium concentration within a negative electrode particle by $c_{\mathrm{a}}^{*}$ and its flux by $\boldsymbol{q}_{\mathrm{a}}^{*}$, while that in a positive electrode particle is denoted by $c_{\mathrm{c}}^{*}$ and its flux by $\boldsymbol{q}_{\mathrm{c}}^{*}$ (the corresponding diffusion coefficients are denoted by $D_{\mathrm{a}}$ and $D_{\mathrm{c}}$, respectively), and write down conservation equations for lithium in the two types of particle:

$\frac{\partial c_{\mathrm{a}}^{*}}{\partial t^{*}}+\nabla^{*} \cdot \boldsymbol{q}_{\mathrm{a}}^{*}=0$ and $\boldsymbol{q}_{\mathrm{a}}^{*}=-D_{\mathrm{a}} \nabla c_{\mathrm{a}}^{*}$ in $\Omega_{\mathrm{a}}$,

$\frac{\partial c_{\mathrm{c}}^{*}}{\partial t^{*}}+\nabla^{*} \cdot \boldsymbol{q}_{\mathrm{c}}^{*}=0$ and $\boldsymbol{q}_{\mathrm{c}}^{*}=-D_{\mathrm{c}} \nabla c_{\mathrm{c}}^{*}$ in $\Omega_{\mathrm{c}}$

assuming that the lithium flux is linearly proportional to its concentration gradient. Other more complicated models have been proposed, for example, in [19] but here we opt for simplicity.

In each electrode, it is necessary to determine the electric potential. In this article, we make the simplest assumptions that the electrode particles are sufficiently well connected (electrically) to the current collectors and sufficiently good conductors so that we can neglect spatial variations in $\phi^{*}$. Without loss of generality, we specify the potential on the anodic (negative) current collector as zero and assume that on the cathodic (positive) current collector as $\Phi^{*}$ so that $\Phi^{*}$ is the potential drop across the whole cell at time $t^{*}$. Denoting $\phi_{\mathrm{a}}^{*}$ and $\phi_{\mathrm{c}}^{*}$ as the potentials in the negative and positive electrodes, respectively; this, together with the above assumptions, leads to conditions of the form:

$\phi_{\mathrm{a}}^{*}=0$ in $\Omega_{\mathrm{a}}$ and $\phi_{\mathrm{c}}^{*}=\Phi^{*}$ in $\Omega_{\mathrm{c}}$.

The electrodelelectrolyte interfaces. In the electrolyte immediately adjacent to the particle interface, it is necessary to account for the behaviour of the Debye layer. It is standard practice to replace the complicated electrochemistry occurring in the Debye layer and on the electrode surface by a number of boundary conditions relating jumps across this narrow layer.

On the surface of the negative electrode (anode) conservation of lithium ions, intercalated lithium and negative ions gives rise to the following conditions on the fluxes there

$\boldsymbol{n} \cdot \boldsymbol{q}_{\mathrm{p}}^{*}=G_{\mathrm{a}}^{*}, \quad \boldsymbol{n} \cdot \boldsymbol{q}_{\mathrm{a}}^{*}=G_{\mathrm{a}}^{*}, \quad \boldsymbol{n} \cdot \boldsymbol{q}_{\mathrm{n}}^{*}=0, \quad$ on $\partial \Omega_{\mathrm{ae}}$,

where, $G_{\mathrm{a}}^{*}$, is the reaction rate of the lithium at the surface. In this article, $G_{\mathrm{a}}^{*}$ depends on the physical conditions on either side of the Debye layer including the potential jump across this layer, the intercalated lithium concentration on the surface of the electrode and the concentration of ions in the electrolyte. The most common form of this is the so-called Butler-Volmer condition (see, e.g. Newman [1, p. 7]). In general the Butler-Volmer condition is given as an empirical law. However, in [26], the three continuity conditions, together with a version of the Butler-Volmer condition, are systematically derived from an analysis of the underlying Poisson-Nernst-Planck (PNP) equations (see, e.g. Newman [1, pp. 271-272]). in the narrow Debye layer and some underlying reactions. For Li-ion cells 
there are a variety of Butler-Volmer relations that are commonly applied. For example, in the case of a negative electrode (anode) composed of $\mathrm{Li}_{x} \mathrm{C}_{6}$, the reaction rate is sometimes expressed in the form [7]:

$$
\begin{aligned}
G_{\mathrm{a}}^{*}=m_{1} c^{* 1 / 2}\left(c_{\mathrm{a}}^{\max }-c_{\mathrm{a}}^{*}\right)^{1 / 2} c_{\mathrm{a}}^{* 1 / 2}[ & \exp \left(-\left(1-\alpha_{\mathrm{a}}\right) \frac{F}{R_{\mathrm{g}} T}\left(\phi^{*}-U^{*}\left(c_{\mathrm{a}}^{*}\right)\right)\right) \\
& \left.-\exp \left(\alpha_{\mathrm{a}} \frac{F}{R_{\mathrm{g}} T}\left(\phi^{*}-U^{*}\left(c_{\mathrm{a}}^{*}\right)\right)\right)\right]\left.\right|_{\partial \Omega_{\mathrm{ae}}},
\end{aligned}
$$

where $\alpha_{\mathrm{a}}$ is the negative electrode (anode) transfer coefficient and lies in the range $0<\alpha_{\mathrm{a}}<1$ (typically it is taken as $1 / 2), m_{1}$ is the reaction rate constant, $c_{\mathrm{a}}^{\max }$ is the maximum possible intercalated lithium concentration in the negative electrode and the function $U^{*}\left(c_{\mathrm{a}}^{*}\right)$ represents the effect of surface lithium ion concentration on the reaction rate (see [7] for an analytic expression for $U^{*}\left(c_{\mathrm{a}}^{*}\right)$ in the case of $\mathrm{Li}_{x} \mathrm{C}_{6}$ which matches to experimental results). Recall that $\phi^{*}$ is the potential in the electrolyte so that potential difference between the electrolyte and the negative electrode is, on referring to (2.4), also $\phi^{*}$.

Similar conditions hold on the positive electrode (cathode) and, denoting the reaction rate by $G_{\mathrm{c}}^{*}$, and these can be written as

$\boldsymbol{n} \cdot \boldsymbol{q}_{\mathrm{p}}^{*}=G_{\mathrm{c}}^{*}, \quad \boldsymbol{n} \cdot \boldsymbol{q}_{\mathrm{c}}^{*}=G_{\mathrm{c}}^{*}, \quad \boldsymbol{n} \cdot \boldsymbol{q}_{\mathrm{n}}^{*}=0, \quad$ on $\partial \Omega_{\mathrm{ce}}$,

where $G_{\mathrm{c}}^{*}$ satisfies a Butler-Volmer equation, for example, for $\mathrm{Li}_{y} \mathrm{Mn}_{2} \mathrm{O}_{4}[5,7,14]$ give

$$
\begin{aligned}
G_{\mathrm{c}}^{*}=m_{2} c^{* 1 / 2}\left(c_{\mathrm{c}}^{\max }-c_{\mathrm{c}}^{*}\right)^{1 / 2} c_{\mathrm{c}}^{* 1 / 2}[ & \exp \left(-\left(1-\alpha_{\mathrm{c}}\right) \frac{F}{R_{\mathrm{g}} T}\left(\phi^{*}-\Phi^{*}-V^{*}\left(c_{\mathrm{c}}^{*}\right)\right)\right) \\
& \left.-\exp \left(\alpha_{\mathrm{c}} \frac{F}{R_{\mathrm{g}} T}\left(\phi^{*}-\Phi^{*}-V^{*}\left(c_{\mathrm{c}}^{*}\right)\right)\right)\right]\left.\right|_{\partial \Omega_{\mathrm{ce}}},
\end{aligned}
$$

where $c_{\mathrm{c}}^{\max }$ is the maximum possible concentration of intercalated lithium in the positive electrode, and $\alpha_{\mathrm{c}}$ is the positive electrode transfer coefficient which is typically taken as $1 / 2$. Here (2.4) implies that $\phi^{*}-\Phi^{*}$ is the potential difference between the electrolyte and the positive electrode. Both the studies [5,7] give analytic expressions for $V^{*}\left(c_{\mathrm{c}}^{*}\right)$ that fit to the experimental data for $\mathrm{Li}_{y} \mathrm{Mn}_{2} \mathrm{O}_{4}$. It is notable that whereas the expressions for $U^{*}\left(c_{\mathrm{a}}^{*}\right)$, in the case of $\mathrm{Li}_{x} \mathrm{C}_{6}$, shows significant variation with changes in $c_{\mathrm{a}}^{*}$ the equivalent expression for $V^{*}\left(c_{\mathrm{c}}^{*}\right)$, in the case of $\mathrm{LiFePO}_{4}$, shows hardly any variation in $c_{\mathrm{c}}^{*}$ throughout most of its range since the discharge curve is almost flat [27]. Further discussion of possible Butler-Volmer conditions can be found in Appendix B.

The external circuit and current collectors. The conditions required to determine $\Phi^{*}$, the potential drop across the cell, depend on the particular external circuit that the battery is connected to. We define the total current, $I$, as the total charge flowing through the cell and hence, due to charge conservation, this can be expressed in terms of an integral of the reaction rates over the surfaces of the two electrodes (i.e. over the total surface of the electrode particles) by

$I^{*}=F \int_{\partial \Omega_{\mathrm{ae}}} G_{\mathrm{a}}^{*} \mathrm{~d} S^{*}$, and $I^{*}=-F \int_{\partial \Omega_{\mathrm{ce}}} G_{\mathrm{c}}^{*} \mathrm{~d} S^{*}$,

where $\mathrm{d} S^{*}$ is an element of surface area. A galvanostatic discharge is where $I^{*}$ is specified and $\Phi^{*}$ is found, but the model can equally be applied to a potentiostatic discharge in which $\Phi^{*}$ is specified and $I^{*}$ is found. Alternatively, if the external circuit behaves as a simple resistor, then Ohm's law holds, and both $I^{*}$ and $\Phi^{*}$ must be found so that $\Phi^{*}=\omega I^{*}$,

where $\omega$ is the resistance of the circuit.

The current collectors are solid, and unreactive, and hence there is no flux of either ion species normal to their interface with the electrolyte. Since the unit vector in $x$-direction, $\boldsymbol{e}_{\boldsymbol{x}}$, is perpendicular to the surface of the collectors, the corresponding boundary conditions are

$\begin{array}{ll}\boldsymbol{q}_{\mathrm{p}}^{*} \cdot \boldsymbol{e}_{\boldsymbol{x}}=\boldsymbol{q}_{\mathrm{n}}^{*} \cdot \boldsymbol{e}_{\boldsymbol{x}}=0 & \text { on } x^{*}=0, \\ \boldsymbol{q}_{\mathrm{p}}^{*} \cdot \boldsymbol{e}_{\boldsymbol{x}}=\boldsymbol{q}_{\mathrm{n}}^{*} \cdot \boldsymbol{e}_{\boldsymbol{x}}=0 & \text { on } x^{*}=\ell .\end{array}$ 
Initial conditions. For simplicity, we assume that the battery has reached an equilibrium state with a certain amount of charge held as intercalated lithium in the particles. Hence, we take ion concentration $c^{*}$ and the intercalated lithium concentration in both negative electrode and positive electrode ( $c_{\mathrm{a}}^{*}$ and $c_{\mathrm{c}}^{*}$, respectively) to be given by

$c^{*}=c^{0},\left.\quad c_{\mathrm{a}}^{*}\right|_{x^{*}<L}=c_{\mathrm{a}}^{0},\left.\quad c_{\mathrm{c}}^{*}\right|_{x^{*}>L}=c_{\mathrm{c}}^{0} \quad$ when $t^{*}=0$.

The porous spacer. We shall consider the porous spacer to be infinitely thin (even on the scale of the particle dimensions), electrically insulating and chemically inert. As a result, we ignore the resistance of the spacer, both to ion transport and to electrical current flow, and as a result, the conditions across this are that the ion concentrations are continuous, the ion fluxes are continuous, and both the electrolyte potential and electric field in the electrolyte are continuous (there is a discontinuity in the electrode particle potential across the separator).

\subsection{Non-dimensionalisation}

The full problem for the electrochemical cell is specified by equations (2.1)-(2.3) and conditions (2.5)-(2.12).

Electrochemical cells are typically constructed such that the distance between the current collectors is small in comparison to the other dimensions of the cell. This observation will, eventually, enable us to write down an approximate one-dimensional averaged model for the battery. However, the electrode microstructure means that the microscale model of the battery, that we consider, must be genuinely three-dimensional. Let us take the area of each of the planar current collectors to be $\mathcal{A}$, denote the magnitude of the typical current by $\bar{I}$ and that of the typical active surface area density (surface area per unit volume) of electrode particles by $\bar{B}$ (this is equivalent to the BET surface area). It follows that the typical current density is $\bar{J}=\bar{I} / \mathcal{A}$ while the size of the electroactive surface area of the negative electrode is of order $\mathcal{A} L \bar{B}$.

Given the geometry of the cell, and our goal of deriving a one-dimensional averaged model for its behaviour, it is helpful to consider the governing equations in a representative section of the cell containing a large number of electrode particles. In particular, it proves necessary to reformulate (2.9) in terms of this section. Taking a representative cylindrical volume through the whole cell of cross-sectional area $L^{2}$, let $\partial \bar{\Omega}_{\mathrm{ae}}^{*}$ be the surface composed of the union of the surfaces of the negative electrode particles within this representative volume. Similarly, we define $\partial \bar{\Omega}_{\mathrm{ce}}^{*}$ to be the surface composed of the union of the surfaces of the positive electrode particles within the representative volume. It follows that (2.9) now takes the form:

$\frac{I^{*} L^{2}}{\mathcal{A}}=F \int_{\partial \bar{\Omega}_{\mathrm{ae}}^{*}} G_{\mathrm{a}}^{*} \mathrm{~d} S^{*}, \quad$ and $\frac{I^{*} L^{2}}{\mathcal{A}}=-F \int_{\partial \bar{\Omega}_{\mathrm{ce}}^{*}} G_{\mathrm{c}}^{*} \mathrm{~d} S^{*}$.

We now non-dimensionalise (2.1)-(2.8) and (2.10)-(2.13) as follows:

$c_{\mathrm{a}}^{*}=c_{\mathrm{s}}^{0} c_{\mathrm{a}}, \quad c_{\mathrm{c}}^{*}=c_{\mathrm{s}}^{0} c_{\mathrm{c}}, \quad c^{*}=c^{0} c, \quad \boldsymbol{x}^{*}=L \boldsymbol{x}, \quad t^{*}=\tau t, \quad \phi^{*}=\bar{\Phi} \phi$,

$I^{*}=\bar{J} \mathcal{A} I, \quad j^{*}=\bar{J} \boldsymbol{j}, \quad G_{\mathrm{a}}^{*}=\frac{\bar{J}}{L \bar{B} F} G_{\mathrm{a}}, \quad G_{\mathrm{c}}^{*}=\frac{\bar{J}}{L \bar{B} F} G_{\mathrm{c}}, \quad \Phi^{*}=\bar{\Phi} \Phi, \quad \boldsymbol{q}_{\mathrm{a}}^{*}=\frac{\bar{J}}{L \bar{B} F} \boldsymbol{q}_{\mathrm{a}}$,

$\boldsymbol{q}_{\mathrm{c}}^{*}=\frac{\bar{J}}{L \bar{B} F} \boldsymbol{q}_{\mathrm{c}}, \quad \boldsymbol{q}_{\mathrm{p}}^{*}=\frac{\bar{J}}{F} \boldsymbol{q}_{\mathrm{p}}, \quad \boldsymbol{q}_{\mathrm{n}}^{*}=\frac{\bar{J}}{F} \boldsymbol{q}_{\mathrm{n}}, \quad U^{*}=\bar{\Phi} U, \quad V^{*}=\bar{\Phi} V$.

Here, $c_{\mathrm{s}}^{0}$ is the typical concentration of intercalated lithium in the electrodes, $\bar{\Phi}$ is the typical potential drop across the cell and $\bar{D}$ is a typical diffusivity in the electrolyte, $\tau$ is the typical timescale for discharge of the cell and areas within the integrals in (2.13) are non-dimensionalised with $L^{2}$. Note that the ion fluxes have been non-dimensionalised with the typical current $\bar{J} \mathcal{A}$ divided by Faraday's constant but that $\boldsymbol{q}_{\mathrm{a}}$ and $\boldsymbol{q}_{\mathrm{c}}$ the fluxes of intercalated lithium in the electrode particles, and the reaction rates at the electrode surface are estimated by dividing the current $\bar{J} \mathcal{A}$ by the typical total surface area of the electrode (i.e. $\mathcal{A} \bar{B} L$ ) multiplied by Faraday's constant. It remains to estimate the timescale $\tau$ for discharge of the cell. An estimate for the volume of the electrode is $L \mathcal{A}$, and it follows that the number of moles of intercalated lithium is of $O\left(c_{\mathrm{s}}^{0} L \mathcal{A}\right)$. The latter are consumed at a rate $\bar{J} \mathcal{A} / F$ and so the typical timescale for discharge of the cell is 
$\tau=\frac{F c_{\mathrm{s}}^{0} L}{\bar{J}}$.

In line with standard practice, we take the transfer coefficients $\alpha_{\mathrm{a}}=1 / 2$ and $\alpha_{\mathrm{c}}=1 / 2$.

On defining the following dimensionless parameters

$\lambda=\frac{F \bar{\Phi}}{R_{\mathrm{g}} T}, \quad \aleph_{\mathrm{a}}=\frac{c_{\mathrm{a}}^{0}}{c_{\mathrm{s}}^{0}}, \quad \aleph_{\mathrm{c}}=\frac{c_{\mathrm{c}}^{0}}{c_{\mathrm{s}}^{0}}, \quad \delta=\frac{1}{\bar{B} L}$,

$\gamma=\frac{F c^{0} \bar{D}}{\bar{J} L}, \quad v=\frac{c_{\mathrm{s}}^{0}}{c^{0}}, \quad \kappa_{\mathrm{p}}=\frac{D_{\mathrm{p}}}{\bar{D}}, \quad \kappa_{\mathrm{n}}=\frac{D_{\mathrm{n}}}{\bar{D}}$,

$\Lambda=\frac{\ell}{L}, \quad \kappa_{\mathrm{a}}=\frac{D_{\mathrm{a}}}{\bar{D}}, \quad \kappa_{\mathrm{c}}=\frac{D_{\mathrm{c}}}{\bar{D}}, \quad \mathcal{C}_{\mathrm{a}}=\frac{c_{\mathrm{a}}^{\max }}{c_{\mathrm{s}}^{0}}$,

$\mathcal{C}_{\mathrm{c}}=\frac{c_{\mathrm{c}}^{\max }}{c_{\mathrm{s}}^{0}}, \quad M_{1}=\frac{m_{1} c_{\mathrm{s}}^{0}\left(c^{0}\right)^{1 / 2} L \bar{B} F}{\bar{J}}, \quad M_{2}=\frac{m_{2} c_{\mathrm{s}}^{0}\left(c^{0}\right)^{1 / 2} L \bar{B} F}{\bar{J}}$,

$K_{1}=\frac{k_{1} c_{\mathrm{s}}^{0} L \bar{B} F}{\bar{J}}, \quad K_{2}=\frac{k_{2} c_{\mathrm{s}}^{0} c^{0} L \bar{B} F}{\bar{J}}, \quad K_{3}=\frac{k_{3} c_{\mathrm{s}}^{0} L \bar{B} F}{\bar{J}}, \quad K_{4}=\frac{k_{4} c_{\mathrm{s}}^{0} c^{0} L \bar{B} F}{\bar{J}}$.

the non-dimensionalisation leads to the following system of dimensionless equations

$\frac{\partial c}{\partial t}+v \nabla \cdot \boldsymbol{q}_{\mathrm{p}}=0, \quad \boldsymbol{q}_{\mathrm{p}}=-\gamma \kappa_{\mathrm{p}}(\nabla c+\lambda c \nabla \phi)$
$\frac{\partial c}{\partial t}+v \nabla \cdot \boldsymbol{q}_{\mathrm{n}}=0, \quad \boldsymbol{q}_{\mathrm{n}}=-\gamma \kappa_{\mathrm{n}}(\nabla c-\lambda c \nabla \phi)$
$\frac{\partial c_{\mathrm{a}}}{\partial t}+\delta \nabla \cdot \boldsymbol{q}_{\mathrm{a}}=0, \quad \boldsymbol{q}_{\mathrm{a}}=-\frac{\gamma \nu}{\delta} \kappa_{\mathrm{a}} \nabla c_{\mathrm{a}} \quad$ in $\tilde{\Omega}_{\mathrm{a}}$,

in $\tilde{\Omega}_{\mathrm{e}}$,

$\boldsymbol{q}_{\mathrm{p}} \cdot \boldsymbol{n}=\delta G_{\mathrm{a}}, \quad \boldsymbol{q}_{\mathrm{n}} \cdot \boldsymbol{n}=0, \quad \boldsymbol{q}_{\mathrm{a}} \cdot \boldsymbol{n}=G_{\mathrm{a}}$,
$\left.G_{\mathrm{a}}=M_{1} c^{1 / 2} c_{\mathrm{a}}^{1 / 2}\left(\mathcal{C}_{\mathrm{a}}-c_{\mathrm{a}}\right)^{1 / 2}\left[\exp \left(-\frac{\lambda}{2}\left(\phi-U\left(c_{\mathrm{a}}\right)\right)\right)-\exp \left(\frac{\lambda}{2}\left(\phi-U\left(c_{\mathrm{a}}\right)\right)\right)\right]\right\} \quad$ on $\partial \tilde{\Omega}_{\mathrm{ae}}$,

$\frac{\partial c_{\mathrm{c}}}{\partial t}+\delta \nabla \cdot \boldsymbol{q}_{\mathrm{c}}=0, \quad \boldsymbol{q}_{\mathrm{c}}=-\frac{\gamma \nu}{\delta} \kappa_{\mathrm{c}} \nabla c_{\mathrm{c}}$ in $\tilde{\Omega}_{\mathrm{c}}$,

$\boldsymbol{q}_{\mathrm{p}} \cdot \boldsymbol{n}=\delta G_{\mathrm{c}}, \quad \boldsymbol{q}_{\mathrm{n}} \cdot \boldsymbol{n}=0, \quad \boldsymbol{q}_{\mathrm{c}} \cdot \boldsymbol{n}=G_{\mathrm{c}}$,

$\left.G_{\mathrm{c}}=M_{2} c^{1 / 2} c_{\mathrm{c}}^{1 / 2}\left(\mathcal{C}_{\mathrm{c}}-c_{\mathrm{c}}\right)^{1 / 2}\left[\exp \left(-\frac{\lambda}{2}\left(\phi-\Phi-V\left(c_{\mathrm{c}}\right)\right)\right)-\exp \left(\frac{\lambda}{2}\left(\phi-\Phi-V\left(c_{\mathrm{c}}\right)\right)\right)\right]\right\}$ on $\partial \tilde{\Omega}_{\mathrm{ce}}$,

$\boldsymbol{q}_{\mathrm{p}} \cdot \boldsymbol{e}_{\boldsymbol{x}}=0, \quad \boldsymbol{q}_{\mathrm{n}} \cdot \boldsymbol{e}_{\boldsymbol{x}}=0, \quad$ on $x=0$,

$\boldsymbol{q}_{\mathrm{p}} \cdot \boldsymbol{e}_{\boldsymbol{x}}=0, \quad \boldsymbol{q}_{\mathrm{n}} \cdot \boldsymbol{e}_{\boldsymbol{x}}=0, \quad$ on $x=\Lambda$,

$I=\delta \int_{\partial \tilde{\Omega}_{\mathrm{ae}}} G_{\mathrm{a}} \mathrm{d} A, \quad I=-\delta \int_{\partial \tilde{\Omega}_{\mathrm{ce}}} G_{\mathrm{c}} \mathrm{d} A$,

$c=1,\left.\quad c_{\mathrm{a}}\right|_{x<1}=\aleph_{\mathrm{a}},\left.\quad c_{\mathrm{c}}\right|_{x>1}=\aleph_{\mathrm{c}}, \quad$ when $t=0$,

and either $I$ specified, or $\Phi$ specified, or $\Phi=\omega I$.

Here, $\partial \tilde{\Omega}_{\mathrm{ae}}$ and $\tilde{\Omega}_{\mathrm{a}}$ are, respectively, the electrode surface and the region occupied by the electrode in a cylinder of unit cross-sectional area with axis between $x=0$ and $x=1 ; \partial \tilde{\Omega}_{\mathrm{ce}}$ and $\tilde{\Omega}_{\mathrm{c}}$ are, respectively, the electrode surface and the region occupied by the electrode contained in a cylinder of unit cross-sectional area between $x=1$ and $x=\Lambda$; In addition, $\tilde{\Omega}_{\mathrm{e}}$ denotes the regions occupied by the electrolyte in a cylinder of unit cross-sectional area with axis between $x=0$ and $x=\Lambda$. It is also useful to write down an expression for the dimensionless current density:

$\boldsymbol{j}=\boldsymbol{q}_{\mathrm{p}}-\boldsymbol{q}_{\mathrm{n}}=-\gamma\left(\left(\kappa_{\mathrm{p}}-\kappa_{\mathrm{n}}\right) \nabla c+\lambda\left(\kappa_{\mathrm{p}}+\kappa_{\mathrm{n}}\right) c \nabla \phi\right)$. 


\subsubsection{Interpretation and discussion of the dimensionless parameters}

In the above equations, $\frac{1}{\delta}$ is a geometric parameter representing the total electrode particle surface area from current collector to current collector per unit cross-sectional area of the cell; $\lambda$ gives the ratio of a typical potential drop across the cell to the thermal voltage; $\kappa_{\mathrm{p}}, \kappa_{\mathrm{n}}, \kappa_{\mathrm{a}}$ and $\kappa_{\mathrm{c}}$ are the dimensionless diffusivities of $\mathrm{Li}^{+}$ions, negative ions and intercalated lithium (in the negative and positive electrodes), respectively; $\gamma$ measures the ratio of the maximum sustainable flux of ions in the electrolyte to the actual ion flux; $\Lambda$ gives the ratio of the total cell width to the width of the negative electrode (anode); $v$ is the ratio of typical intercalated lithium concentrations in the solid to that in the electrolyte; and $M_{1}$ and $M_{2}$ are dimensionless reaction rates.

We have chosen to non-dimensionalise so that the dimensionless fluxes $\boldsymbol{q}_{\mathrm{n}}, \boldsymbol{q}_{\mathrm{p}}, \boldsymbol{q}_{\mathrm{a}}$ and $\boldsymbol{q}_{\mathrm{c}}$ are typically of magnitude 1 . In addition, we note that the dimensionless parameter $\lambda$ will play a particularly important role in the analysis that follows. The thermal voltage $R_{\mathrm{g}} T / F$ is, at room temperature of about $25 \mathrm{mV}$ and so $\lambda$ will be large in most applications. Furthermore, we remark that the factors $\delta$ appearing in equations $(2.16 \mathrm{~g})$ ensure that the right-hand sides of these equations are of magnitude 1 , the dimensionless surface areas of $\partial \Omega_{\mathrm{ae}}$ and $\partial \Omega_{\mathrm{ce}}$ being of magnitude $1 / \delta$.

We can also use existing data to estimate the size of the other important dimensional parameters in the problem

$$
\begin{aligned}
& a \approx 10^{-5} \mathrm{~m}, \quad D_{\mathrm{p}} \approx 10^{-11}-10^{-10} \mathrm{~m}^{2} \mathrm{~s}^{-1}, \quad D_{\mathrm{n}} \approx \frac{D_{\mathrm{p}}}{3} \mathrm{~m}^{2} \mathrm{~s}^{-1}, \quad D_{\mathrm{a}} \text { and } D_{\mathrm{c}} \approx 10^{-16}-10^{-13} \mathrm{~m}^{2} \mathrm{~s}^{-1}, \\
& c^{0} \approx 10^{3} \mathrm{molm}^{-3}, \quad c_{\mathrm{s}}^{0} \approx 50 \times 10^{3} \mathrm{molm}^{-3}, \quad L \approx 1.5 \times 10^{-4} \mathrm{~m},
\end{aligned}
$$

where $a$ gives a typical radius of an electrode particle and the figures for $D_{\mathrm{p}}$ and $D_{\mathrm{n}}$, are based on conductivities of lithium ion salts [28]. The figures for solid diffusion of intercalated lithium $\left(D_{\mathrm{a}}\right.$ and $\left.D_{\mathrm{c}}\right)$ are taken from data on lithium-metal-manganese oxides $D_{\mathrm{c}} \sim 10^{-16}-10^{-13} \mathrm{~m}^{2} \mathrm{~s}^{-1}$ [29], lithium-tungsten-oxide $D_{\mathrm{c}} \sim$ $2 \times 10^{-15}-6 \times 10^{-15} \mathrm{~m}^{2} \mathrm{~s}^{-1}$ [30], lithium cobalt oxide $D_{\mathrm{c}} \sim 10^{-14}-1.5 \times 10^{-13} \mathrm{~m}^{2} \mathrm{~s}^{-1}$ [31] and lithium-graphite $D_{\mathrm{a}} \sim 10^{-16}-10^{-13} \mathrm{~m}^{2} \mathrm{~s}^{-1}$ [32]. We now use these dimensional parameters to estimate the key dimensionless parameters:

$\lambda \approx 180, \kappa_{\mathrm{p}}=1, \kappa_{\mathrm{n}} \approx 1 / 3, \kappa_{\mathrm{a}}$ and $\kappa_{\mathrm{c}} \approx 10^{-1}-10^{-6}, v \approx 50$.

We have little information about the sizes of the dimensionless reaction constants $K_{1}, K_{2}, K_{3}$ and $K_{4}$. However, as long as their sizes are not excessively large or small, this makes little difference since the exponentials in (2.16c)(2.16e) contain the large parameter $\lambda$ and will thus be dominant. Finally, we can estimate $\delta$ by considering the electrode to be a collection of uniform spheres of radius $a$ in a number of possible packing scenarios. The packing makes little difference to the size of $\delta$ so, on using the information above to deduce that $a / L$ is typically about $1 / 15$, it follows that

$\delta \approx 2 \times 10^{-2}-5 \times 10^{-2}$.

In this study, we shall assume that the diffusion of material through the electrode particles occurs over a shorter timescale than diffusion through the electrolyte (which is thus the limiting process). In terms of the above dimensionless parameters this is, as we shall show, equivalent to the assumption that $\gamma \nu \kappa_{\mathrm{a}} \gg \delta^{2}$ and $\gamma \nu \kappa_{\mathrm{a}} \gg \delta^{2}$ (which holds for at least some part of the parameter range).

\subsection{Alternative forms for equations and boundary conditions}

In the following analysis, it is worth noting that (2.16a) can always be rearranged to give an equation solely in terms of $c$ and further manipulations allow $\phi$ to be eliminated from the boundary conditions $(2.16 \mathrm{c}(\mathrm{I}))-(2.16 \mathrm{c}(\mathrm{II}))$ and $(2.16 \mathrm{e}(\mathrm{I}))-(2.16 \mathrm{e}(\mathrm{II}))$. The result is

$$
\begin{aligned}
& \frac{\partial c}{\partial t}=2 \gamma \nu \frac{\kappa_{\mathrm{n}} \kappa_{\mathrm{p}}}{\kappa_{\mathrm{n}}+\kappa_{\mathrm{p}}} \nabla^{2} c \text { in } \tilde{\Omega}_{\mathrm{e}}, \\
& \left.\frac{\partial c}{\partial n}\right|_{\partial \tilde{\Omega}_{\mathrm{ae}}}=-\frac{\delta G_{\mathrm{a}}}{2 \gamma \kappa_{\mathrm{p}}},\left.\quad \frac{\partial c}{\partial n}\right|_{\partial \tilde{\Omega}_{\mathrm{ce}}}=-\frac{\delta G_{\mathrm{c}}}{2 \gamma \kappa_{\mathrm{p}}} .
\end{aligned}
$$


Besides, if we know the reaction rates, $G_{\mathrm{a}}$ and $G_{\mathrm{c}}$, this provides a closed system for $c$ in $\tilde{\Omega}_{\mathrm{e}}$. In a similar way, we can find equations and boundary conditions for $\phi$ to give

$$
\begin{aligned}
& \nabla \cdot(c \nabla \phi)=\frac{1}{\lambda} \frac{\kappa_{\mathrm{n}}-\kappa_{\mathrm{p}}}{\kappa_{\mathrm{n}}+\kappa_{\mathrm{p}}} \nabla^{2} c, \\
& \left.c \frac{\partial \phi}{\partial n}\right|_{\partial \tilde{\Omega}_{\mathrm{ae}}}=-\frac{\delta G_{\mathrm{a}}}{2 \lambda \gamma \kappa_{\mathrm{p}}},\left.\quad c \frac{\partial \phi}{\partial n}\right|_{\partial \tilde{\Omega}_{\mathrm{ce}}}=-\frac{\delta G_{\mathrm{c}}}{2 \lambda \gamma \kappa_{\mathrm{p}}} .
\end{aligned}
$$

\section{Multiple-scale formalism}

The multiple-scale technique [33-35] gives a systematic method for accounting for phenomena that occur within a microscopic quasiperiodic microstructure in terms of a macroscopic system of equations. In particular, it provides a method for deriving the macroscopic equations and determining the corresponding (macroscopic) physical parameters from a local problem for the microscopic behaviour. This local problem exploits the quasiperiodic structure to allow the microscale solution to be formally averaged and thus accounts for the fine geometric details and properties of the material. We will apply this method to the microscale battery model (2.16b)-(2.16i) and (2.18)-(2.21) to derive a macroscopic electrode scale model. This proves to be very similar to the macroscopic models formulated and used in $[2,4,5,7,8]$. The novelty of this study is that it provides a systematic method for calibrating the coefficients in the model in terms of the microstructure and thereby provides a tool for improving particle design.

This approach can be contrasted with averaging methods see, for example, Wang et al. [36] where spatial averages are taken of the microscopic equations resulting in equations on a macroscopic scale for the microscopically averaged variables. These macroscopic equations are closed by making ad hoc assumptions about higher-order averages (the closure conditions) and fitting these to empirical data. In this study, we exploit the large difference in lengthscale between the electrode particles and the battery thickness, as denoted by $\delta$, to use an asymptotic multiple-scale method to systematically approximate the problem. This allows for deriving equations governing the macroscopic behaviour, but formally relies on the particle packing being nearly periodic. The method has the considerable advantage over the averaging method in that it allows parameters in the macroscopic problem to be derived directly, from the underlying microscopic geometry and physical model, without resorting to ad hoc closure conditions. Given that manufacturers are now producing electrode particles to high tolerance, this approach gives a method for systematically investigating the effect of different particle designs on the performance of the battery.

The limit $\delta \rightarrow 0$ will be taken to allow a multi-scale analysis (for example [33-35]), to homogenise the equations and create a simplified macroscopic model.

The above method assumes that the solution is a function of two disparate lengthscales corresponding to that of the electrode particles $(O(\delta)$ in dimensionless units) and that of the electrolytic cell $(O(1)$ in dimensionless units). In order to analyse the problem, it is helpful to introduce a microscale variable $\hat{\boldsymbol{x}}$ on the particle lengthscale. Furthermore, we assume that there is local periodicity in the electrode particle arrangement and, in particular, that the structure is periodic within a repeating domain $\hat{V}_{\text {per }} \cup \hat{\Omega}_{\text {per }}$ (see Fig. 3). In fact, in order to keep the treatment as generalised as possible, we shall allow the shape and size of the particles (denoted by $\hat{\Omega}_{\text {per }}$ ) to vary over the long lengthscale $\boldsymbol{x}$ but, as a consequence of the multiple-scale method we employ, we require that the union of the domain occupied by the particles $\hat{\Omega}_{\text {per }}$ and that occupied by the electrolyte lying outside them $\hat{V}_{\text {per }}$ to be strictly periodic. Rather than rescale $\boldsymbol{x}$ with $\delta$ directly, we rescale with $\epsilon$ where $\epsilon=\delta p$ measures the size of the periodic domain $\hat{V}_{\text {per }} \cup \hat{\Omega}_{\text {per }}$ (e.g. the length of its edges where it is cuboidal) and $p$ is an $O$ (1) parameter. Thus the microscale variable is defined by

$\boldsymbol{x}=\epsilon \hat{\boldsymbol{x}}$,

where $\hat{\boldsymbol{x}}$ measures distances on the scale of an electrode particle and $\boldsymbol{x}$ measures them on the scale of the electrode. In the standard fashion we now look for a solution which is a function both of the long-scale variable $\boldsymbol{x}$ and of the short scale variable $\hat{\boldsymbol{x}}$. This will lead to a non-uniquely determined problem unless we impose some extra conditions. 


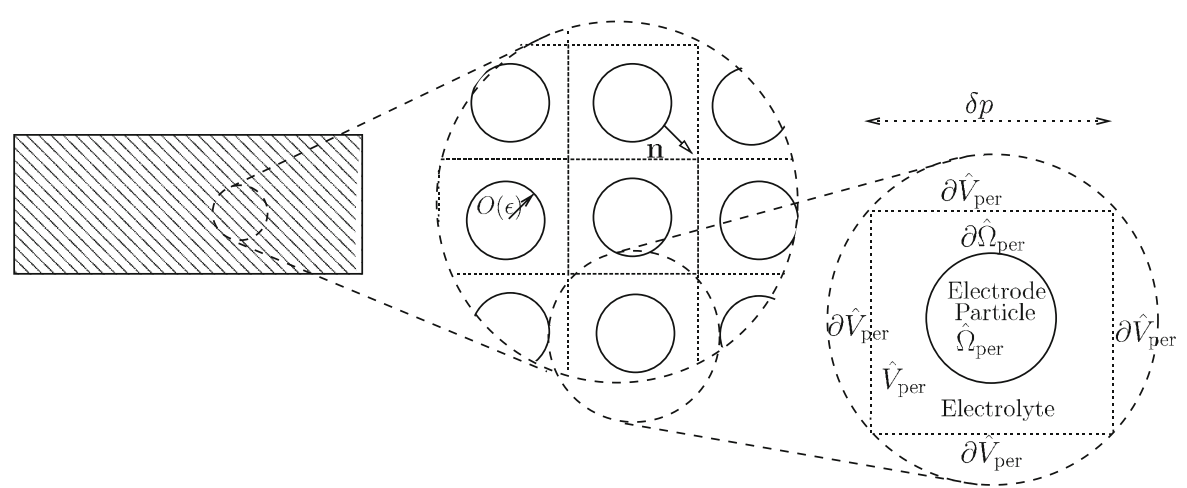

Fig. 3 Illustration of the microstructured boundary

Here we require that the solution is periodic in the short-scale variable. Having made this solution ansatz, it becomes apparent that the gradient operator transforms according to $\nabla \rightarrow \nabla+\hat{\nabla} / \epsilon$.

The central ansatz for the expansions of the variables is

$$
\begin{aligned}
& c=c_{0}(\boldsymbol{x}, t)+\epsilon c_{1}(\hat{\boldsymbol{x}}, \boldsymbol{x}, t)+\epsilon^{2} c_{2}(\hat{\boldsymbol{x}}, \boldsymbol{x} t)+\ldots, \\
& \phi=\phi_{0}(\boldsymbol{x}, t)+\epsilon \phi_{1}(\hat{\boldsymbol{x}}, \boldsymbol{x}, t)+\epsilon^{2} \phi_{2}(\hat{\boldsymbol{x}}, \boldsymbol{x} t)+\ldots, \\
& \boldsymbol{q}_{\mathrm{p}}=\boldsymbol{q}_{p, 0}(\hat{\boldsymbol{x}}, \boldsymbol{x}, t)+\epsilon \boldsymbol{q}_{p, 1}(\hat{\boldsymbol{x}}, \boldsymbol{x}, t)+\epsilon^{2} \boldsymbol{q}_{p, 2}(\hat{\boldsymbol{x}}, \boldsymbol{x}, t)+\ldots, \\
& \boldsymbol{q}_{\mathrm{n}}=\boldsymbol{q}_{n, 0}(\hat{\boldsymbol{x}}, \boldsymbol{x}, t)+\epsilon \boldsymbol{q}_{n, 1}(\hat{\boldsymbol{x}}, \boldsymbol{x}, t)+\epsilon^{2} \boldsymbol{q}_{n, 2}(\hat{\boldsymbol{x}}, \boldsymbol{x}, t)+\ldots, \\
& \boldsymbol{j}=\boldsymbol{j}_{0}(\hat{\boldsymbol{x}}, \boldsymbol{x}, t)+\epsilon \boldsymbol{j}_{1}(\hat{\boldsymbol{x}}, \boldsymbol{x}, t)+\epsilon^{2} \boldsymbol{j}_{2}(\hat{\boldsymbol{x}}, \boldsymbol{x}, t)+\ldots,
\end{aligned}
$$

so that both the concentration and the potential in the electrolyte are dominated by variations on the battery lengthscale with important smaller variations on the electrode-particle lengthscale. The fluxes $\boldsymbol{q}_{\mathrm{p}}$ and $\boldsymbol{q}_{\mathrm{n}}$ and the current density $\boldsymbol{j}$ all have to flow around the electrode particles and hence display significant variations on the particle lengthscale. It is clear that averaged quantities for the lithium ion concentration and the potential are given by $\langle c\rangle(\boldsymbol{x}, t) \sim c_{0}(\boldsymbol{x}, t)$ and $\langle\phi\rangle(\boldsymbol{x}, t) \sim \phi_{0}(\boldsymbol{x}, t)$, respectively. However, it is not immediately clear how to define the average current density $\langle\boldsymbol{j}\rangle(\boldsymbol{x}, t)$, for example.

\subsection{Homogenisation in the electrolyte}

The method of multiple scales is used in Appendix A to write down an averaged equation for the diffusion of lithium ions in the electrolyte and the potential in the electrolyte. By comparing (2.18)-(2.21) with (7.1)-(7.3), we can identify

$D=2 \gamma \nu \frac{\kappa_{\mathrm{n}} \kappa_{\mathrm{p}}}{\kappa_{\mathrm{n}}+\kappa_{\mathrm{p}}}, \quad \epsilon=\delta p, \quad k=\frac{\nu \kappa_{\mathrm{n}}}{p\left(\kappa_{\mathrm{n}}+\kappa_{\mathrm{p}}\right)}, \quad \alpha=\frac{\kappa_{\mathrm{n}}-\kappa_{\mathrm{p}}}{\kappa_{\mathrm{n}}+\kappa_{\mathrm{p}}}$.

Then it follows, by analogy with (7.24) and (7.25), that the equations for $c_{0}(\boldsymbol{x}, t)$ and $\phi_{0}(\boldsymbol{x}, t)$ are

$$
\begin{aligned}
& \frac{\left|\hat{V}_{\mathrm{per}}\right|}{\left|\hat{V}_{\mathrm{per}}\right|+\left|\hat{\Omega}_{\mathrm{per}}\right|} \frac{\partial c}{\partial t}-2 \gamma \nu \frac{\kappa_{\mathrm{n}} \kappa_{\mathrm{p}}}{\kappa_{\mathrm{n}}+\kappa_{\mathrm{p}}} \frac{\partial}{\partial x_{i}}\left(\mathcal{B}_{i j} \frac{\partial c}{\partial x_{j}}\right)=\frac{\nu \kappa_{\mathrm{n}}}{p\left(\kappa_{\mathrm{n}}+\kappa_{\mathrm{p}}\right)} \tilde{Q}, \\
& \frac{\partial}{\partial x_{i}}\left(\mathcal{B}_{i j}\left(\lambda c \frac{\partial \phi}{\partial x_{j}}-\left(\frac{\kappa_{\mathrm{n}}-\kappa_{\mathrm{p}}}{\kappa_{\mathrm{n}}+\kappa_{\mathrm{p}}}\right) \frac{\partial c}{\partial x_{j}}\right)\right)+\frac{\tilde{Q}}{p \gamma\left(\kappa_{\mathrm{n}}+\kappa_{\mathrm{p}}\right)}=0 .
\end{aligned}
$$

Here, we drop the subscripts from $c_{0}$ and $\phi_{0}$, define the diffusion tensor $\mathcal{B}_{i j}$ in (7.19), define the effective reaction rates, $\tilde{Q}, \tilde{G}_{\mathrm{a}}$ and $\tilde{G}_{\mathrm{c}}$ by 
$\tilde{Q}=\left\{\begin{array}{l}\tilde{G}_{\mathrm{a}}=\frac{\int_{\partial \hat{\Omega}_{\mathrm{per}}} G_{\mathrm{a}} \mathrm{d} \hat{S}}{\left|\hat{\Omega}_{\mathrm{per}}\right|+\left|\hat{V}_{\mathrm{per}}\right|} \text { in the negative electrode } \\ \tilde{G}_{\mathrm{c}}=\frac{\int_{\partial \hat{\Omega}_{\mathrm{per}}} G_{\mathrm{c}} \mathrm{d} \hat{S}}{\left|\hat{\Omega}_{\mathrm{per}}\right|+\left|\hat{V}_{\mathrm{per}}\right|} \text { in the positive electrode }\end{array}\right.$

and introduce the geometric parameters $\left|\hat{\Omega}_{\text {per }}\right|=\int_{\hat{\Omega}_{\text {per }}} \mathrm{d} \hat{V}$ and $\left|\hat{V}_{\text {per }}\right|=\int_{\hat{V}_{\text {per }}} \mathrm{d} \hat{V}$. In Sect. 7.2, we prove that the tensor $\mathcal{B}_{i j}$ is symmetric and that all its diagonal entries are smaller than 1 (implying that the diffusivity is reduced by the microstructure)

$\mathcal{B}_{i j}=\mathcal{B}_{j i}, \quad 0<\mathcal{B}_{i i}<1 \quad$ (no summation).

The tensor $\mathcal{B}_{i j}$ is a multiple of the effective diffusivity tensor usually introduced in macroscopic models. Here, the definition (7.19) gives a prescriptive method for determining these values for any given microscopic geometry and set of physical parameters. The reduction of the effective diffusivity in any particular direction below the value of the diffusivity without any microstructure can be attributed to tortuosity. The quantity $\tilde{Q}$ represents the averaged fluxes into and out of the electrolyte due to transfer between particles and electrolyte.

\subsection{Behaviour in the electrode particles}

As stated previously we consider the fast diffusion limit in which the concentration of lithium $c_{\mathrm{a}}$ and $c_{\mathrm{c}}$, in both negative and positive electrodes, is, to a good approximation, independent of position within the particle. Conservation of lithium within a particle implies that the rate at which lithium concentration changes within the particle, multiplied by the volume of the particle, is equal to the rate at which it is extracted from the particle surface so that

$$
\begin{aligned}
\int_{\hat{\Omega}_{\mathrm{per}}} \frac{\partial c_{\mathrm{a}}}{\partial t} \mathrm{~d} \hat{V} & =-\frac{1}{p} \int_{\partial \hat{\Omega}_{\mathrm{per}}} G_{\mathrm{a}} \mathrm{d} \hat{S} \Longrightarrow \frac{\left|\hat{\Omega}_{\mathrm{per}}\right|}{\left|\hat{\Omega}_{\mathrm{per}}\right|+\left|\hat{V}_{\mathrm{per}}\right|} \frac{\partial c_{\mathrm{a}}}{\partial t}=-\frac{1}{p} \tilde{G}_{\mathrm{a}}, \\
\int_{\hat{\Omega}_{\mathrm{per}}} \frac{\partial c_{\mathrm{c}}}{\partial t} \mathrm{~d} \hat{V} & =-\frac{1}{p} \int_{\partial \hat{\Omega}_{\mathrm{per}}} G_{\mathrm{c}} \mathrm{d} \hat{S} \Longrightarrow \frac{\left|\hat{\Omega}_{\mathrm{per}}\right|}{\left|\hat{\Omega}_{\mathrm{per}}\right|+\left|\hat{V}_{\mathrm{per}}\right|} \frac{\partial c_{\mathrm{c}}}{\partial t}=-\frac{1}{p} \tilde{G}_{\mathrm{c}},
\end{aligned}
$$

where $\tilde{G}_{\mathrm{a}}$ and $\tilde{G}_{\mathrm{c}}$ are defined by (3.5). Equally, we could have derived these equations by performing a formal asymptotic expansion of $c_{\mathrm{a}}$ and $c_{\mathrm{c}}$ in powers of $\delta^{2} / \gamma \nu \kappa_{\mathrm{a}}$ and $\delta^{2} / \gamma \nu \kappa_{\mathrm{a}}$, respectively, using (2.16b) and (2.16d).

3.3 The transfer rates at the interfaces between particles and electrolyte

Consider now the equations relating the current to the reaction rates $(2.16 \mathrm{~g})$. The volume of each microscale periodic domain $\hat{V}_{\text {per }} \cup \hat{\Omega}_{\text {per }}$ on the microscale is $(\delta p)^{3}\left(\int_{\hat{V}_{\text {per }}} \mathrm{d} \hat{V}+\int_{\hat{\Omega}_{\text {per }}} \mathrm{d} \hat{V}\right)$, that is $(\delta p)^{3}\left(\left|\hat{\Omega}_{\text {per }}\right|+\left|\hat{V}_{\text {per }}\right|\right)$. The number of microscale periodic domains contained within a macroscopic volume $\mathrm{d} V$ is thus $\mathrm{d} V /\left((\delta p)^{3}\left(\left|\hat{\Omega}_{\mathrm{per}}\right|+\left|\hat{V}_{\text {per }}\right|\right)\right)$ and furthermore $\mathrm{d} A=(\delta p)^{2} \mathrm{~d} \hat{S}$, where $\mathrm{d} \hat{S}$ is an element of surface area on the electrode particle lengthscale. It is known that $\partial \tilde{\Omega}_{\mathrm{ae}}$ is the electroactive surface contained in a cylinder of unit cross-sectional area between $x=0$ and $x=1$ while $\partial \tilde{\Omega}_{\mathrm{ce}}$ is the electroactive surface contained in a cylinder of unit cross-sectional area between $x=1$ and $x=\Lambda$ (so that the element of volume in both these cases is $\mathrm{d} V=\mathrm{d} x$ ) it follows that

$\int_{\partial \tilde{\Omega}_{\mathrm{ae}}} G_{\mathrm{a}} \mathrm{d} A=\int_{0}^{1}(\delta p)^{2}\left(\int_{\partial \hat{\Omega}_{\mathrm{per}}} G_{\mathrm{a}} \mathrm{d} \hat{S}\right) \frac{\mathrm{d} x}{(\delta p)^{3}\left(\left|\hat{\Omega}_{\mathrm{per}}\right|+\left|\hat{V}_{\mathrm{per}}\right|\right)}$, 


$$
\int_{\partial \tilde{\Omega}_{\mathrm{ce}}} G_{\mathrm{c}} \mathrm{d} A=\int_{1}^{\Lambda}(\delta p)^{2}\left(\int_{\partial \hat{\Omega}_{\mathrm{per}}} G_{\mathrm{c}} \mathrm{d} \hat{S}\right) \frac{\mathrm{d} x}{(\delta p)^{3}\left(\left|\hat{\Omega}_{\mathrm{per}}\right|+\left|\hat{V}_{\mathrm{per}}\right|\right)} .
$$

In turn this can be rewritten as

$$
\int_{\partial \tilde{\Omega}_{\mathrm{ae}}} G_{\mathrm{a}} \mathrm{d} A=\frac{1}{\delta p} \int_{0}^{1} \tilde{G}_{\mathrm{a}}(x) \mathrm{d} x \int_{\partial \tilde{\Omega}_{\mathrm{ce}}} G_{\mathrm{c}} \mathrm{d} A=\frac{1}{\delta p} \int_{1}^{\Lambda} \tilde{G}_{\mathrm{c}}(x) \mathrm{d} x .
$$

Hence, we can rewrite $(2.16 \mathrm{~g})$ in terms of the locally averaged reaction rates $\tilde{G}_{\mathrm{a}}$ and $\tilde{G}_{\mathrm{c}}$ as

$I=\frac{1}{p} \int_{0}^{1} \tilde{G}_{\mathrm{a}}(x) \mathrm{d} x, \quad I=-\frac{1}{p} \int_{1}^{\Lambda} \tilde{G}_{\mathrm{c}}(x) \mathrm{d} x$.

\subsection{Summary of the homogenised equations}

On applying the definition (3.5) to (2.16c), and (2.16e) (recalling that $c_{\mathrm{a}}, c_{\mathrm{c}}, c$ and $\phi$ are all approximately constant over a particle) and on dropping subscripts from (2.16f), (3.3)-(3.4), (3.6) and (3.7) we can write the homogenised model as

$$
\begin{aligned}
& \frac{\left|\hat{V}_{\mathrm{per}}\right|}{\left|\hat{\Omega}_{\mathrm{per}}\right|+\left|\hat{V}_{\mathrm{per}}\right|} \frac{\partial c}{\partial t}-2 \gamma \nu \frac{\kappa_{\mathrm{n}} \kappa_{\mathrm{p}}}{\kappa_{\mathrm{n}}+\kappa_{\mathrm{p}}} \frac{\partial}{\partial x_{i}}\left(\mathcal{B}_{i j} \frac{\partial c}{\partial x_{j}}\right)=\left\{\begin{array}{ll}
\frac{\nu \kappa_{\mathrm{n}}}{p\left(\kappa_{\mathrm{n}}+\kappa_{\mathrm{p}}\right)} \tilde{G}_{\mathrm{a}} & \text { in } 0<x<1 \\
\frac{\nu \kappa_{\mathrm{n}}}{p\left(\kappa_{\mathrm{n}}+\kappa_{\mathrm{p}}\right)} \tilde{G}_{\mathrm{c}} & \text { in } 1<x<\Lambda
\end{array},\right. \\
& \frac{\partial}{\partial x_{i}}\left(\mathcal{B}_{i j}\left(\lambda c \frac{\partial \phi}{\partial x_{j}}-\left(\frac{\kappa_{\mathrm{n}}-\kappa_{\mathrm{p}}}{\kappa_{\mathrm{n}}+\kappa_{\mathrm{p}}}\right) \frac{\partial c}{\partial x_{j}}\right)\right)=\left\{\begin{array}{ll}
-\frac{\tilde{G}_{\mathrm{a}}}{p \gamma\left(\kappa_{\mathrm{n}}+\kappa_{\mathrm{p}}\right)} & \text { in } 0<x<1 \\
-\frac{\tilde{G}_{\mathrm{c}}}{p \gamma\left(\kappa_{\mathrm{n}}+\kappa_{\mathrm{p}}\right)} & \text { in } 1<x<\Lambda
\end{array},\right. \\
& \tilde{G}_{\mathrm{a}}=\tilde{M}_{1} c_{\mathrm{a}}^{1 / 2}\left(\mathcal{C}_{\mathrm{a}}-c_{\mathrm{a}}\right)^{1 / 2} c^{1 / 2}\left[\exp \left(-\frac{\lambda}{2}\left(\phi-U\left(c_{\mathrm{a}}\right)\right)\right)-\exp \left(\frac{\lambda}{2}\left(\phi-U\left(c_{\mathrm{a}}\right)\right)\right)\right], \\
& \tilde{G}_{\mathrm{c}}=\tilde{M}_{2} c_{\mathrm{c}}^{1 / 2}\left(\mathcal{C}_{\mathrm{c}}-c_{\mathrm{c}}\right)^{1 / 2} c^{1 / 2}\left[\exp \left(-\frac{\lambda}{2}\left(\phi-\Phi-V\left(c_{\mathrm{c}}\right)\right)\right)-\exp \left(\frac{\lambda}{2}\left(\phi-\Phi-V\left(c_{\mathrm{c}}\right)\right)\right)\right], \\
& \frac{\left|\hat{\Omega}_{\text {per }}\right|}{\left|\hat{\Omega}_{\text {per }}\right|+\left|\hat{V}_{\text {per }}\right|} \frac{\partial c_{\mathrm{a}}}{\partial t}=-\frac{1}{p} \tilde{G}_{\text {a }} \text { in } 0<x<1, \\
& \frac{\left|\hat{\Omega}_{\mathrm{per}}\right|}{\left|\hat{\Omega}_{\mathrm{per}}\right|+\left|\hat{V}_{\mathrm{per}}\right|} \frac{\partial c_{\mathrm{c}}}{\partial t}=-\frac{1}{p} \tilde{G}_{\mathrm{c}} \text { in } 1<x<\Lambda, \\
& I=\frac{1}{p} \int_{0}^{1} \tilde{G}_{\mathrm{a}}(x) \mathrm{d} x, \quad I=-\frac{1}{p} \int_{1}^{\Lambda} \tilde{G}_{\mathrm{c}}(x) \mathrm{d} x, \\
& \left.c\right|_{t=0}=1,\left.\quad c_{\mathrm{a}}\right|_{x<1, t=0}=\aleph_{\mathrm{a}},\left.\quad c_{\mathrm{c}}\right|_{x>\Lambda, t=0}=\aleph_{\mathrm{c}},
\end{aligned}
$$




$$
\left.\frac{\partial c}{\partial x}\right|_{x=0}=0,\left.\quad \frac{\partial \phi}{\partial x}\right|_{x=0}=0,\left.\quad \frac{\partial c}{\partial x}\right|_{x=\Lambda}=0,\left.\quad \frac{\partial \phi}{\partial x}\right|_{x=\Lambda}=0,
$$

where $c_{\mathrm{a}}$ is the intercalated lithium concentration in the negative electrode, $c_{\mathrm{c}}$ is that in the positive electrode and

$\tilde{M}_{1}=M_{1} \frac{\int_{\partial \hat{\Omega}_{\mathrm{per}}} \mathrm{d} \hat{S}}{\left|\hat{\Omega}_{\mathrm{per}}\right|+\left|\hat{V}_{\mathrm{per}}\right|}, \quad \tilde{M}_{2}=M_{2} \frac{\int_{\partial \hat{\Omega}_{\mathrm{per}}} \mathrm{d} \hat{S}}{\left|\hat{\Omega}_{\mathrm{per}}\right|+\left|\hat{V}_{\text {per }}\right|}$.

\subsubsection{Comparison to other models in the literature and redimensionalisation of the homogenised equations}

This system of equations is similar to the averaged macroscopic models used elsewhere $[2,4,5,7,8]$. One important difference to these models is that here we have assumed that the electrical conductivity between particles is very good so that the conductivity in the electrodes is effectively infinite. ${ }^{1}$ In order to further highlight this similarity between the model that we have derived here (3.8a)-(3.8i) and those used elsewhere, we identify the average current density in the electrolyte $\langle\boldsymbol{j}\rangle$ and rewrite $(3.8 \mathrm{~b})$ in terms of $\langle\boldsymbol{j}\rangle$

$\langle\boldsymbol{j}\rangle_{i}=-\gamma \mathcal{B}_{i j}\left(\left(\kappa_{\mathrm{p}}-\kappa_{\mathrm{n}}\right) \frac{\partial c}{\partial x_{j}}+\lambda c \frac{\partial \phi}{\partial x_{j}}\right), \quad \nabla \cdot\langle\boldsymbol{j}\rangle=\left\{\begin{array}{ll}\frac{\tilde{G}_{\mathrm{a}}}{p} & \text { in } 0<x<1 \\ \frac{\tilde{G}_{\mathrm{c}}}{p} & \text { in } 1<x<\Lambda\end{array}\right.$.

Here, to the level of approximation used in deriving the averaged equations, $c=\langle c\rangle$ and $\phi=\langle\phi\rangle$. In terms of the notation used in [7], the (dimensionless) averaged electrolyte current $\boldsymbol{i}_{\mathrm{e}}=\langle\boldsymbol{j}\rangle$, the (dimensionless) transfer current per unit volume is $S_{\mathrm{av}} j_{\mathrm{n}}=\tilde{G}_{\mathrm{a}} / p$ in the negative electrode and $S_{\mathrm{av}} j_{\mathrm{n}}=\tilde{G}_{\mathrm{c}} / p$ in the positive electrode, where $S_{\mathrm{av}}$ is the average electroactive area. It is notable that our derivation of the model gives a systematic method for deriving the macroscopic electrolyte diffusion tensor $\mathcal{B}_{i j}$ and further that the effective conductivity of the electrolyte is uniquely defined in terms of this diffusion tensor. The method we have used therefore has the considerable advantage over the standard averaging techniques of determining ad hoc fitting parameters in the macroscopic model uniquely from the underlying microscopic model. If these parameters are inconsistent with data, then this implies that the microscopic model provides an inadequate description of the underlying physical processes.

In order to make a more detailed comparison to other models, we redimensionalise (3.8a)-(3.8b), using (2.14)(2.15), obtaining

$$
\begin{aligned}
& \varepsilon_{\mathrm{v}} \frac{\partial c^{*}}{\partial t^{*}}-\frac{\partial}{\partial x_{i}}\left(\frac{2 D_{\mathrm{n}} D_{\mathrm{p}}}{D_{\mathrm{n}}+D_{\mathrm{p}}} \mathcal{B}_{i j} \frac{\partial c^{*}}{\partial x_{j}^{*}}\right)=\frac{D_{\mathrm{n}}}{D_{\mathrm{n}}+D_{\mathrm{p}}} a^{*} G^{*}, \\
& \nabla^{*} \cdot\left\langle\boldsymbol{j}^{*}\right\rangle=-F a^{*} G^{*}, \\
& \left\langle\boldsymbol{j}^{*}\right\rangle_{\mathrm{p}}=-\left(\frac{\left(D_{\mathrm{n}}+D_{\mathrm{p}}\right) F^{2} c^{*}}{R T}\right) \mathcal{B}_{\mathrm{pq}}\left(\frac{\partial \phi^{*}}{\partial x_{\mathrm{q}}^{*}}-\frac{R T}{F}\left(\frac{D_{\mathrm{n}}-D_{\mathrm{p}}}{D_{\mathrm{n}}+D_{\mathrm{p}}}\right) \frac{\partial}{\partial x_{\mathrm{q}}^{*}} \log c^{*}\right),
\end{aligned}
$$

where $a^{*}$, the BET surface area, and $\varepsilon_{\mathrm{v}}$, the particle volume fraction are defined by

$a^{*}=\frac{1}{\delta p L} \frac{\int_{\partial \hat{\Omega}_{\mathrm{per}}} \mathrm{d} \hat{S}}{\left|\hat{\Omega}_{\mathrm{per}}\right|+\left|\hat{V}_{\mathrm{per}}\right|}, \quad \varepsilon_{\mathrm{v}}=\frac{\left|\hat{V}_{\mathrm{per}}\right|}{\left|\hat{\Omega}_{\mathrm{per}}\right|+\left|\hat{V}_{\mathrm{per}}\right|}$

and $G^{*}=G_{\mathrm{a}}^{*}$ in the negative electrode and $G^{*}=G_{\mathrm{c}}^{*}$ in the positive electrode. Rewriting this in the form used by Fuller, Doyle and Newman [7] gives

$$
\begin{aligned}
& \varepsilon_{\mathrm{v}} \frac{\partial c^{*}}{\partial t^{*}}-\frac{\partial}{\partial x_{i}}\left(\varepsilon_{\mathrm{v}} \mathcal{D}_{i j} \frac{\partial c^{*}}{\partial x_{j}^{*}}\right)=\left(1-t_{+}\right) a^{*} G^{*}, \\
& \nabla^{*} \cdot\left\langle\boldsymbol{j}^{*}\right\rangle=F a^{*} G^{*}, \\
& \left\langle\boldsymbol{j}^{*}\right\rangle_{\mathrm{p}}=-\kappa_{\mathrm{pq}}\left(\frac{\partial \phi^{*}}{\partial x_{\mathrm{q}}^{*}}-\frac{R T}{F}\left(1-2 t_{+}\right) \frac{\partial}{\partial x_{\mathrm{q}}^{*}} \log c^{*}\right),
\end{aligned}
$$

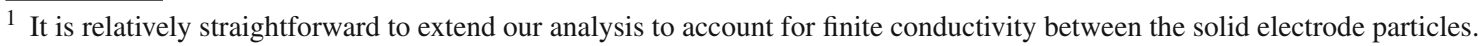


where the transference number of lithium $t_{+}$, the conductivity tensor $\kappa_{i j}$ and the effective diffusivity $\mathcal{D}_{i j}$ are given by

$t_{+}=\frac{D_{\mathrm{p}}}{D_{\mathrm{n}}+D_{\mathrm{p}}}, \quad \kappa_{i j}=\frac{F^{2}\left(D_{\mathrm{n}}+D_{\mathrm{p}}\right)}{R T} c^{*} \mathcal{B}_{i j}, \quad \mathcal{D}_{i j}=\frac{2 R T}{F^{2} \varepsilon_{\mathrm{v}} c^{*}} t_{+}\left(1-t_{+}\right) \kappa_{i j}$.

In terms of the notation used in [7] we can identify $G^{*}$ with $j_{\mathrm{n}},\left\langle\boldsymbol{j}^{*}\right\rangle$ with $\boldsymbol{i}_{2}$ and $\phi^{*}$ with $\Phi_{2}$. Note that the factor $\left(1-2 t_{+}\right)$appearing in (3.16) differs from that given in [7] where the factor is given as $\left(1-t_{+}\right)$. This difference comes about because [7] uses the concentrated solution theory described in [1], in contrast to this study where we make use of a dilute theory for which the Stokes-Einstein relation relating diffusivity to mobility is applicable. This difference between the two theories leads to differences in the definitions of electrolyte diffusivities, ion mobilities and transference numbers. Indeed, with our definition of the transference number, replacing the factor $\left(1-2 t_{+}\right)$ with $\left(1-t_{+}\right)$in $(3.16)$ is incorrect as can be seen using symmetry considerations.

In the next section, physically relevant limiting cases of the remaining parameters in the problem are considered to identify the underlying behaviour of the battery.

\section{Asymptotic analysis $(\lambda \gg 1)$}

Here, we consider a one-dimensional problem for the homogenised model describing a planar cell in which the negative electrode occupies the region $0<x<1$ while the positive electrode occupies the region $1<x<\Lambda$ and is separated from the negative electrode by a thin spacer. We will only investigate the case where the open circuit potential curves $U\left(c_{\mathrm{a}}\right)$ and $V\left(c_{\mathrm{c}}\right)$ both show significant variations with $c_{\mathrm{a}}$ and $c_{\mathrm{c}}$, respectively. We note that where the open circuit potential curves are so flat that $O(1)$ variations in $c_{\mathrm{a}}$ and $c_{\mathrm{c}}$ lead to variations in $U\left(c_{\mathrm{a}}\right)$ and $V\left(c_{\mathrm{c}}\right)$ of size $1 / \lambda$ the asymptotic analysis we present here is inappropriate and a more complicated analysis is required (thus the results we present here could not be utilised to describe an $\mathrm{LiFePO}_{4}$ electrode, for example). Furthermore, we shall assume that the composition of the electrodes is uniform within each electrode. As we are considering one-dimensional behaviour in the $x$-direction, we need only to consider $\mathcal{B}_{11}$ and we assume it to have the form:

$$
\begin{aligned}
& \mathcal{B}_{11}=\left(\mathcal{B}_{-} H(1-x)+\mathcal{B}_{+} H(x-1)\right), \quad\left|\hat{\Omega}_{\text {per }}\right|=\left|\hat{\Omega}_{\mathrm{a}}\right| H(1-x)+\left|\hat{\Omega}_{\mathrm{c}}\right| H(x-1), \\
& \quad \text { and }\left|\hat{V}_{\text {per }}\right|=\left|\hat{V}_{\mathrm{a}}\right| H(1-x)+\left|\hat{V}_{\mathrm{c}}\right| H(x-1)
\end{aligned}
$$

where $\left|\hat{\Omega}_{\mathrm{a}}\right|,\left|\hat{\Omega}_{\mathrm{c}}\right|,\left|\hat{V}_{\mathrm{a}}\right|$ and $\left|\hat{V}_{\mathrm{c}}\right|$ are all constants and $H(\cdot)$ is the usual Heaviside function. The constants $\mathcal{B}_{-}$and $\mathcal{B}_{+}$can be calculated for any particular microstructure in the positive and negative electrodes using the methods in Appendix A. We note that the particle volume fractions $\left|\hat{\Omega}_{\mathrm{a}}\right| /\left(\left|\hat{V}_{\mathrm{a}}\right|+\left|\hat{\Omega}_{\mathrm{a}}\right|\right)$ and $\left|\hat{\Omega}_{\mathrm{c}}\right| /\left(\left|\hat{V}_{\mathrm{c}}\right|+\left|\hat{\Omega}_{\mathrm{c}}\right|\right)$ are well known for various packings of spherical particles (being, e.g. 0.74 for face centred cubic and 0.52 for simple cubic).

We base our asymptotic analysis of the model on the assumptions that $\lambda \gg 1$ and, in line with parameter estimates, considering the distinguished limit $\gamma=O(1), \kappa_{\mathrm{n}}=O(1), \kappa_{\mathrm{p}}=O(1)$ and $v=O(\lambda)$. From a physical point of view, $\gamma=O(1)$ corresponds to placing a very high load on the battery and so is a particularly interesting limit. Following the assumptions made about the sizes of the parameter $v$, we write

$\nu=\lambda \tilde{\nu}$,

where $\tilde{v}$ is a $O(1)$ parameter. The assumption that $\gamma \nu$ is large corresponds to the timescale for diffusion within the electrolyte being much faster than that for the reaction in the electrode particles so that the first term in (3.8a) is negligible.

We assume that the variables have a regular expansion in $1 / \lambda$ as follows:

$$
\begin{aligned}
& c=c_{0}(x, t)+\ldots, \quad \phi=\phi_{0}(t)+\frac{1}{\lambda} \phi_{1}(x, t)+\ldots, \quad \Phi=\Phi_{0}+\frac{1}{\lambda} \Phi_{1} \ldots, \\
& c_{\mathrm{a}}=c_{\mathrm{a}, 0}+\frac{1}{\lambda} c_{\mathrm{a}, 1}+\ldots, \quad c_{\mathrm{c}}=c_{\mathrm{c}, 0}+\frac{1}{\lambda} c_{\mathrm{c}, 1}+\ldots, \quad G_{\mathrm{a}}=G_{\mathrm{a} 0}+\frac{1}{\lambda} G_{\mathrm{a}, 1}+\ldots, \\
& G_{\mathrm{c}}=G_{\mathrm{c} 0}+\frac{1}{\lambda} G_{\mathrm{a}, 1}+\ldots, \quad \tilde{G}_{\mathrm{a}}=\tilde{G}_{\mathrm{a} 0}+\frac{1}{\lambda} \tilde{G}_{\mathrm{a} 1}+\ldots, \quad \tilde{G}_{\mathrm{c}}=\tilde{G}_{\mathrm{c} 0}+\frac{1}{\lambda} \tilde{G}_{\mathrm{c} 1}+\ldots
\end{aligned}
$$


The electrolyte. Substitution of the above expansion into (3.8a), (3.8i(I)) and (3.8i(III)) yields

$$
\begin{aligned}
& \frac{\partial}{\partial x}\left(\left(\mathcal{B}_{-} H(1-x)+\mathcal{B}_{+} H(x-1)\right) \frac{\partial c_{0}}{\partial x}\right)+\frac{1}{2 p \gamma \kappa_{\mathrm{p}}}\left(\tilde{G}_{\mathrm{a} 0} H(1-x)+\tilde{G}_{\mathrm{c} 0} H(x-1)\right)=0, \\
& \left.\frac{\partial c_{0}}{\partial x}\right|_{x=0}=0,\left.\quad \frac{\partial c_{0}}{\partial x}\right|_{x=\Lambda}=0 .
\end{aligned}
$$

Since the highest derivative in $t$ does not appear in the leading order equation for $c$ (because $v \gg 1$ ), there is an initial short timescale transient in which the solution rapidly adjusts to a quasi-steady state. We do not wish to investigate this transient, but nevertheless the solution to the Neumann problem (4.3) and (4.4) is not currently uniquely defined, and so we require an extra condition which comes from matching to this transient behaviour. In this case, we can avoid much analysis by noting that the problem conserves the global ion concentration. It is apparent from (3.8a) that there is no net consumption of lithium ions, implying that the average lithium ion concentration across the cell is independent of time. Since $c$ has been non-dimensionalised with average initial ion concentration, this implies

$\int_{0}^{\Lambda} c_{0} \mathrm{~d} x=\Lambda$

and this condition, when applied to (4.3) and (4.4) should, where a solution for $c_{0}$ exists, determine it uniquely.

The discontinuity in $\mathcal{B}$ at $x=1$ means that, when looking for the solution to (4.4), we should impose the jump conditions $\left[c_{0}\right]_{x=1^{-}}^{x=1^{+}}=0$ and $\left[\mathcal{B} \partial c_{0} / \partial x\right]_{x=1^{-}}^{x=1^{+}}=0$ in the standard fashion.

Butler-Volmer conditions. Substitution of the Butler-Volmer conditions (3.8c) and (3.8d) into (3.8e) and (3.8f) leads to evolution equations for $c_{\mathrm{a}}$ and $c_{\mathrm{c}}$ with exponential decay, which rapidly approach a steady solution. This occurs because both $U\left(c_{\mathrm{a}}\right)$ and $V\left(c_{\mathrm{c}}\right)$ are monotonically increasing in their arguments (corresponding to the difference in potentials between the electrolyte and the electrode increasing with electrode lithium concentration). To leading order, this steady solution is found by setting the arguments of the exponentials in the Butler-Volmer conditions (3.8c)-(3.8d) to zero so that

$\phi_{0}(t)=U\left(c_{\mathrm{a}, 0}\right), \quad \Phi_{0}=\phi_{0}(t)-V\left(c_{\mathrm{c}, 0}\right)$.

Variations in the electrolyte potential are the size of the thermal voltage, which is much smaller than the drop across the battery (in these units $O(1 / \lambda)$ compared to $O(1)$ ). Figure 1 shows a typical plot of the potentials in the battery. Where these conditions are satisfied, the relation between the lithium concentrations in the electrodes $\left(c_{\mathrm{a}}\right.$ and $\left.c_{\mathrm{c}}\right)$, the electrolyte potential $\phi$ and the potential drop across the cell $\Phi$ are given, at leading order, by the two expression in (4.6). An important corollary to this is that both the leading order lithium concentrations in the electrode particles $\left(c_{\mathrm{a}, 0}\right.$ and $\left.c_{\mathrm{c}, 0}\right)$ are functions of time only. A noteworthy feature of this solution is that the potential drop across the cell $\Phi$ is, to the lowest order of approximation, independent of the current $I$, being drawn from the cell and only dependent on the state of discharge of the cell. This potential drop is typically termed the OCV to denote that is the same as the voltage that would be obtained after sufficient time at open circuit.

The electrode particles. We can infer from the facts that $c_{\mathrm{a}, 0}=c_{\mathrm{a}, 0}(t)$ and $c_{\mathrm{c}, 0}=c_{\mathrm{c}, 0}(t)$ and from the leading order expansion of (3.8e) and (3.8f) that

$\frac{\partial \tilde{G}_{\mathrm{a} 0}}{\partial x}=0, \quad \frac{\partial \tilde{G}_{\mathrm{c} 0}}{\partial x}=0$

(recall that $\left|\hat{\Omega}_{\text {per }}\right|$ and $\left|\hat{V}_{\text {per }}\right|$ are both constant within each electrode). It follows from (3.8g) that the discharge is spatially uniform with

$\tilde{G}_{\mathrm{a} 0}=p I, \quad \tilde{G}_{\mathrm{c} 0}=-\frac{p I}{\Lambda-1}$.

Equations for the evolution of $c_{\mathrm{a}, 0}$ and $c_{\mathrm{c}, 0}$ arise from substitution of the above into the leading terms of (3.8e) and (3.8f) and are

$\frac{\mathrm{d} c_{\mathrm{a}, 0}}{\mathrm{~d} t}=-I\left(\frac{\left|\hat{V}_{\mathrm{a}}\right|+\left|\hat{\Omega}_{\mathrm{a}}\right|}{\left|\hat{\Omega}_{\mathrm{a}}\right|}\right), \quad \frac{\mathrm{d} c_{\mathrm{c}, 0}}{\mathrm{~d} t}=\frac{I}{(\Lambda-1)}\left(\frac{\left|\hat{V}_{\mathrm{c}}\right|+\left|\hat{\Omega}_{\mathrm{c}}\right|}{\left|\hat{\Omega}_{\mathrm{c}}\right|}\right)$. 
Integrating (4.9) and applying the initial conditions $\left.c_{\mathrm{a}, 0}\right|_{t=0}=\aleph_{\mathrm{a}}$ and $\left.c_{\mathrm{c}, 0}\right|_{t=0}=\aleph_{\mathrm{c}}$ gives expressions for the lithium concentrations in the electrodes

$$
c_{\mathrm{a}, 0}(t)=\aleph_{\mathrm{a}}-\left(\frac{\left|\hat{V}_{\mathrm{a}}\right|+\left|\hat{\Omega}_{\mathrm{a}}\right|}{\left|\hat{\Omega}_{\mathrm{a}}\right|}\right) \int_{0}^{t} I \mathrm{~d} t, \quad c_{\mathrm{c}, 0}(t)=\aleph_{\mathrm{c}}+\frac{1}{\Lambda-1}\left(\frac{\left|\hat{V}_{\mathrm{c}}\right|+\left|\hat{\Omega}_{\mathrm{c}}\right|}{\left|\hat{\Omega}_{\mathrm{c}}\right|}\right) \int_{0}^{t} I \mathrm{~d} t .
$$

\subsection{Behaviour for currents below $I_{\text {crit }}$}

We start by considering the solution for the concentration of electrolyte. We can integrate (4.3)-(4.5), where the reaction rates $\tilde{G}_{\mathrm{a} 0}$ and $\tilde{G}_{\mathrm{c} 0}$ are given by (4.8), to obtain a solution for $c_{0}$ of the form:

$c_{0}=\left\{\begin{array}{l}1+\frac{I}{12 \Lambda \gamma \kappa_{\mathrm{p}}}\left(\frac{2(\Lambda-1)^{2}}{\mathcal{B}_{+}}+\frac{3 \Lambda-2-3 \Lambda x^{2}}{\mathcal{B}_{-}}\right) \text {in } 0<x<1 \\ 1+\frac{I}{12 \Lambda \gamma \kappa_{\mathrm{p}}}\left(\frac{3 \Lambda(\Lambda-x)^{2}}{\mathcal{B}_{+}(\Lambda-1)}-\frac{\Lambda^{2}+\Lambda-2}{\mathcal{B}_{+}}-\frac{2}{\mathcal{B}_{-}}\right) \quad \text { in } 1<x<\Lambda\end{array}\right.$.

Typical plots of this solution are made in Figs. $4 \mathrm{a}$ and $5 \mathrm{a}$. One of its notable features is that for sufficiently large $I$, the concentrations can become negative and therefore unphysical. In this section, we therefore consider the case where the concentration remains positive. For simplicity, we focus on the case $I>0$, corresponding to battery discharge, in which $c_{0}$ decreases monotonically in $x$ with minimum at $x=\Lambda$ and we see that (4.11) remains strictly positive where

$I<I_{\text {crit }} \quad$ with $I_{\text {crit }}=\frac{12 \gamma \kappa_{\mathrm{p}} \Lambda \mathcal{B}_{+} \mathcal{B}_{-}}{\mathcal{B}_{-}\left(\Lambda^{2}+\Lambda-2\right)+2 \mathcal{B}_{+}}$.

It is interesting to compare our results with those of Fuller et al. [7] for their model system which suggest a critical current density, in dimensional terms, of about $4.0 \mathrm{~mA} / \mathrm{cm}^{2}$. Using their data and the above formula leads to value of the critical current density of about $9.0 \mathrm{~mA} / \mathrm{cm}^{2}$. The reason for the difference in these values is that while the analysis we present here is applicable to $\mathrm{Li}_{x} \mathrm{C}_{6}$, in that the utilization of lithium within this material is almost uniform across the negative electrode (Fig. 7 of [7]), it is not applicable for the $\mathrm{Li}_{y} \mathrm{Mn}_{2} \mathrm{O}_{4}$ in the positive electrode which has an open circuit potential with several very flat sections to it (Fig. A-2 of [7]) and consequently does not discharge uniformly across the electrode (Fig. 7 of [7]). The asymptotic description of materials with such flat open circuit potentials is beyond the scope of this article, but will be tackled by the authors in a future study.

The correction to the open circuit voltage. In Appendix $\mathrm{C}$, we calculate the $O(1 / \lambda)$ correction to the $\mathrm{OCV}$ when $I<I_{\text {crit }}$. This is dependent on the exact form of the Butler-Volmer condition used. We find that, for example, when we use the conditions (3.8c)-(3.8d) that

$\Phi_{1}=-\int_{0}^{1} \log c_{0}(x, t)+2 \log \mathcal{G}(x, t) \mathrm{d} x+\frac{1}{\Lambda-1} \int_{1}^{\Lambda} \log c_{0}(x, t)+2 \log \mathcal{H}(x, t) \mathrm{d} x$,

where

$\mathcal{G}=\frac{p I+\left(p^{2} I^{2}+4 \tilde{M}_{1}^{2} c_{0} c_{\mathrm{a}, 0}\left(\mathcal{C}_{\mathrm{a}}-c_{\mathrm{a}, 0}\right)\right)^{1 / 2}}{2 \tilde{M}_{1} c_{\mathrm{a}, 0}^{1 / 2} c_{0}^{1 / 2}\left(\mathcal{C}_{\mathrm{a}}-c_{\mathrm{a}, 0}\right)^{1 / 2}}, \quad \mathcal{H}=\frac{-p I+\left(p^{2} I^{2}+4(\Lambda-1)^{2} \tilde{M}_{2}^{2} c_{0} c_{\mathrm{c}, 0}\left(\mathcal{C}_{\mathrm{c}}-c_{\mathrm{c}, 0}\right)\right)^{1 / 2}}{2 \tilde{M}_{2}(\Lambda-1) c_{\mathrm{c}, 0}^{1 / 2} c_{0}^{1 / 2}\left(\mathcal{C}_{\mathrm{c}}-c_{\mathrm{c}, 0}\right)^{1 / 2}}$.

Hence, if the galvanostatic current is less than the critical value $I_{\text {crit }}$, then the electrolyte has significant amounts of lithium throughout the battery and, to the lowest order, the discharge is uniform. The dominant behaviour of the concentration and the potential is independent of the particular Butler-Volmer condition that was considered. However, there are details, as shown by the corrections, that are dependent on the model that is exploited. Such details may indicate the appropriate model that is required to be used. 

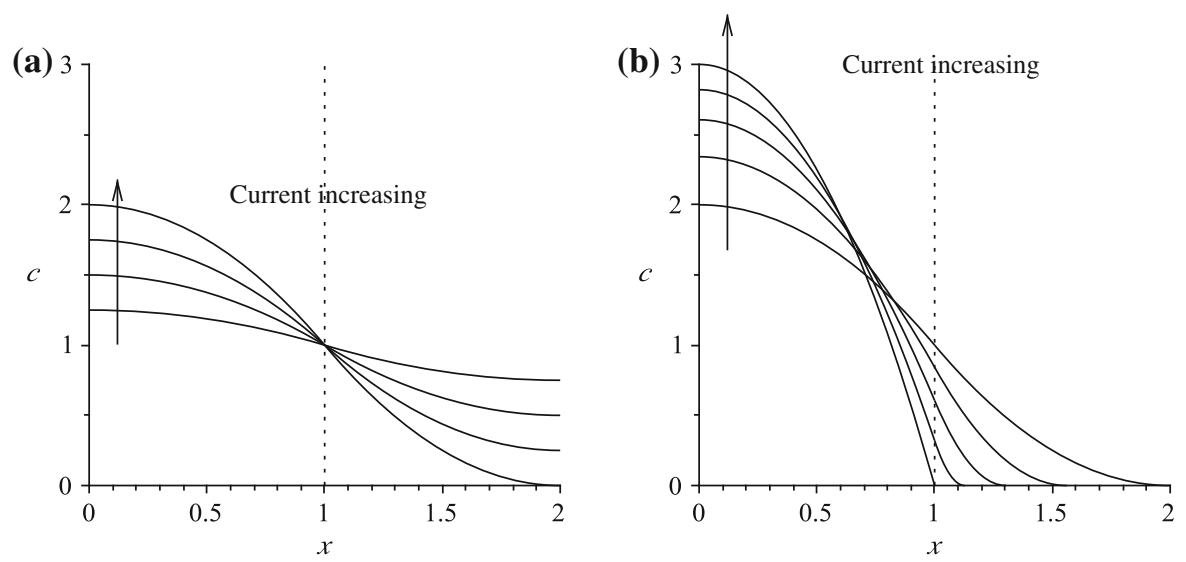

Fig. 4 Plots of the solution to (4.3)-(4.4) for $\Lambda=2, \mathcal{B}_{+}=1, \mathcal{B}_{-}=1$ for which $I_{\text {crit }}=4 \gamma \kappa_{\mathrm{p}}$ and $I_{\max }=12 \gamma \kappa_{\mathrm{p}}$. a The current takes the values $I / I_{\text {crit }}=0.25,0.5,0.75,1$ and, since the current is low enough to allow significant reaction throughout both electrodes, the solution for $c_{0}$ is regular (of form (4.11)). b The currents are greater than $I_{\text {crit }}$, taking the values $I / I_{\text {crit }}=1,1.5,2,2.5,3$, and the solution consequently has the form (4.21) in which regions of the positive electrode are unable to discharge properly
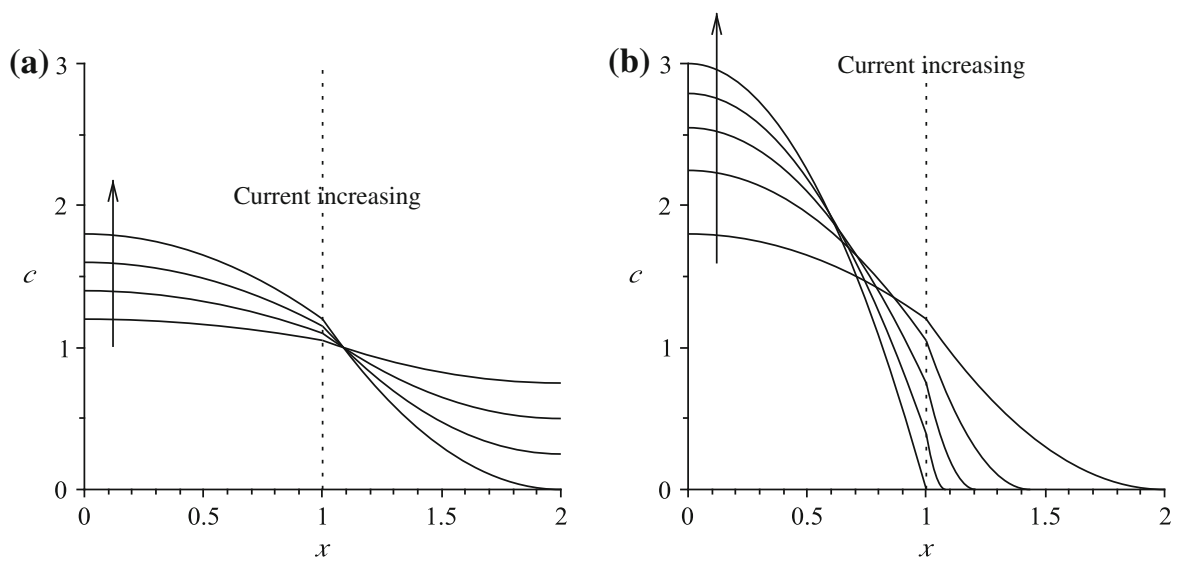

Fig. 5 Plots of the solution to (4.3)-(4.4) for $\Lambda=2, \mathcal{B}_{+}=1, \mathcal{B}_{-}=2$ for which $I_{\text {crit }}=4.8 \gamma \kappa_{\mathrm{p}}$ and $I_{\max }=24 \gamma \kappa_{\mathrm{p}}$. a The current takes the values $I / I_{\text {crit }}=0.25,0.5,0.75,1$ and the solution for $c_{0}$ is regular (of form (4.11)). b The current takes the values $I / I_{\text {crit }}=1,2,3,4,5$ and the solution has the form (4.21)

\subsection{A free boundary solution for electrolyte ion concentration at high currents $\left(I>I_{\text {crit }}\right)$}

We now return to the question of whether a physical solution exists when the current is greater than $I_{\text {crit }}$. Physically, it can be seen that (4.11) breaks down for large $I$ because of the inability of ions to diffuse sufficiently rapidly towards the ion-depleted region of the negative electrode. Since the diffusion timescale is highly dependent on distance over which diffusion takes place, it might be expected that, at high currents, the regions of the negative electrode farthest from the separator will become depleted of lithium in the electrolyte and effectively switch off. Since the total current is specified, this will result in increased reaction rates in parts of the electrode close to the separator.

To simplify our examination of behaviour, we shall only consider positive $I$ so that electrolyte depletion occurs within the positive electrode in $1<x<\Lambda$; the analysis is similar for $I<0$ except that the electrolyte-depleted region lies in $0<x<1$. From a physical point of view, it is clear that the problem occurs because the reaction on the electrode is so fast that it significantly depletes the electrolyte within the positive electrode leaving regions far from the separator unable to react because of the lack of available lithium ions. 
Fig. 6 A schematic of $\mathrm{Li}^{+}$ concentration $c_{0}$ at high current $I>I_{\text {crit }}$

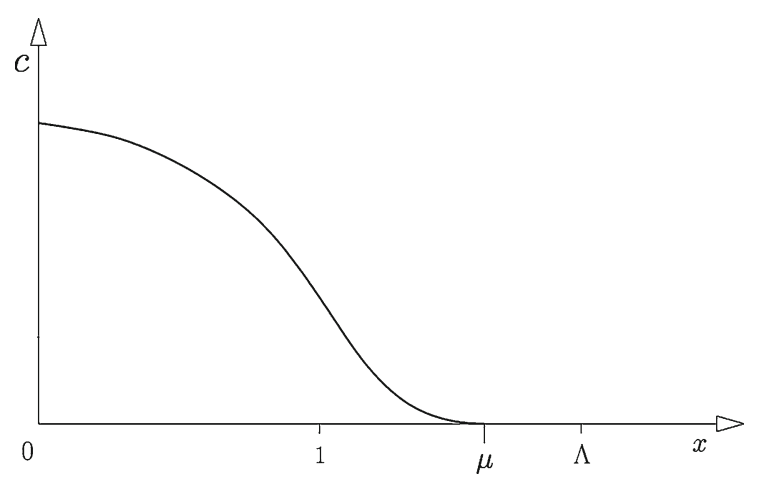

We shall study this problem more carefully in Appendix D but to demonstrate the general behaviour while avoiding much of the detail, we consider introducing a free boundary at $x=\mu(t)$ (where $1<\mu<\Lambda$ ) to describe the solution. This boundary divides the region with significant lithium-ion concentration, close to the separator $(1<x<\mu(t))$, from a region, far from the separator, where the lithium concentration in the electrolyte is extremely small $\mu(t)<x<\Lambda$ as illustrated in Fig. 6. We will consider the region $\mu(t)<x<\Lambda$, where $c_{0}=0$ at the end of this section, wherein, in reality, the ion concentrations are exponentially small and first we concentrate of the region $1<x<\mu(t)$. In this region $c_{0}=O(1)$ and at the free boundary, we expect

$c_{0}=0$ and $\frac{\partial c_{0}}{\partial x}=0$ on $x=\mu$.

representing the facts that ion concentration vanishes (to leading order) on the free boundary and that the flux of ions into the ion-depleted region is negligible. Hence, the problem is to find the concentration of lithium in the electrolyte and find the position of a free boundary where the concentration goes to zero.

\subsubsection{Region of significant ion concentration $0<x<\mu$}

In performing the asymptotic analysis, we should expand all the variables as before but also expand $\mu(t)$. Once again since, $\phi_{0}$ is a function of $t$ only, the leading order Butler-Volmer conditions

$$
\begin{aligned}
& \phi_{0}(t)=U\left(c_{\mathrm{a}, 0}\right) \quad \text { in } 0<x<1, \\
& \Phi_{0}(t)=\phi_{0}(t)-V\left(c_{\mathrm{c}, 0}\right) \quad \text { in } 1<x<\mu(t),
\end{aligned}
$$

imply that $c_{\mathrm{a}, 0}$ and $c_{\mathrm{c}, 0}$ are functions of time only. It follows from the integral conditions $(3.8 \mathrm{~g})$ that

$\tilde{G}_{\mathrm{a}, 0}=p I \quad$ in $0<x<1, \quad$ and $\quad \tilde{G}_{\mathrm{c}, 0}= \begin{cases}-\frac{p I}{\mu-1} & \text { in } 1<x<\mu \\ 0 & \text { in } \mu<x<\Lambda\end{cases}$

Substituting these expressions for the reaction rates into (3.8a), (3.8h(I)), (3.8i(I)), (4.5) and (4.14) gives the following system for $c$ at leading order:

$$
\begin{aligned}
& 2 \kappa_{\mathrm{p}} \frac{\partial}{\partial x}\left(\left(\mathcal{B}_{-} H(1-x)+\mathcal{B}_{+} H(x-1)\right) \frac{\partial c_{0}}{\partial x}\right)= \begin{cases}-\frac{I}{\gamma} & \text { in } 0<x<1, \\
\frac{I}{\gamma(\mu-1)} \text { in } 1<x<\mu\end{cases} \\
& \left.\frac{\partial c_{0}}{\partial x}\right|_{x=0}=0,\left.\quad \frac{\partial c_{0}}{\partial x}\right|_{x=\mu}=0,\left.\quad c_{0}\right|_{x=\mu}=0, \\
& \int_{0}^{\mu} c_{0} \mathrm{~d} x=\Lambda,
\end{aligned}
$$


while substituting them into (3.8e), (3.8f), (3.8h(II)-(III)) gives the following equations for the evolution of the lithium concentrations in the electrodes (to leading order):

$$
\begin{aligned}
& \frac{\partial c_{\mathrm{a}, 0}}{\partial t}=-\frac{\left|\hat{V}_{\mathrm{a}}\right|+\left|\hat{\Omega}_{\mathrm{a}}\right|}{\left|\hat{\Omega}_{\mathrm{a}}\right|} I \text { in } 0<x<1, \\
& \frac{\partial c_{\mathrm{c}, 0}}{\partial t}= \begin{cases}\frac{\left|\hat{V}_{\mathrm{c}}\right|+\left|\hat{\Omega}_{\mathrm{c}}\right|}{\left|\hat{\Omega}_{\mathrm{c}}\right|} \frac{I}{\mu-1} & \text { in } 1<x<\mu, \\
0 & \text { in } \mu<x\end{cases}
\end{aligned}
$$

We note that (4.16)-(4.18) appears overdetermined but since the integral of the right-hand side of (4.16) is zero, the condition (4.16b) is a consequence of (4.16a). Solving (4.16)-(4.17) gives

$c_{0}=\left\{\begin{array}{ll}\frac{I}{4 \gamma \kappa_{\mathrm{p}}}\left(\frac{1}{\mathcal{B}_{-}}+\frac{\mu-1}{\mathcal{B}_{+}}-\frac{x^{2}}{\mathcal{B}_{-}}\right) & \text {in } 0<x<1 \\ \frac{I}{4 \gamma \kappa_{\mathrm{p}}} \frac{(x-\mu)^{2}}{\mathcal{B}_{+}(\mu-1)} & \text { in } 1<x<\mu\end{array}\right.$.

Applying the integral condition (4.18) to this solution leads to a quadratic for $\mu$

$(\mu-1)^{2}+3(\mu-1)+\left(\frac{2 \mathcal{B}_{+}}{\mathcal{B}_{-}}-\frac{12 \mathcal{B}_{+} \gamma \kappa_{\mathrm{p}} \Lambda}{I}\right)=0$,

which we must solve, subject to the requirement that $1<\mu<\Lambda$, to determine the position of the free boundary $x=\mu$. Plots of $c_{0}$ are made for different values of the current in Fig. 4. These plots show that as the current $I$ is increased, the free-boundary moves back towards the separator at $x=1$.

In order for a solution $\mu$ to (4.22) to lie in the required range $1<x<\lambda$, the following conditions must be satisfied by the current:

$I_{\text {crit }}<I<I_{\max } \quad\left(\right.$ with $\left.I_{\max }=6 \mathcal{B}_{-} \gamma \kappa_{\mathrm{p}} \Lambda\right)$

If the current $I$ is less than $I_{\text {crit }}$, then the solution for $c_{0}$ has no free boundary, ionic concentrations are significant everywhere and the analysis of Sect. 4.1 is appropriate; here the solution is approximated by (4.11). For $I$ in the range $I_{\text {crit }}<I<I_{\max }$, the analysis of this section is valid. However, this study indicates that if $I>I_{\max }$, then no solution exists because there is insufficient electrolyte to carry current across the negative electrode.

Remark. The free boundary solution that we have generated is for $I>0$; however, this can readily be modified to the case $I<0$ by having the free boundary in the negative electrode (i.e. in $0<x<1$ ).

If we consider the lowest order behaviour of galvanostatic discharge of a battery, then for small currents, we have found that all parts of the battery participate in this behaviour and the discharge is spatially uniform. For current greater than $I_{\text {crit }}$, there are parts of the battery where the lithium in the electrolyte is depleted to the point where these no longer participate in the reaction, and hence there are spatial variations in the use of the stored lithium. There is a larger current $I_{\max }$ at which one electrode of the battery is completely depleted. We speculate that in practice, when the current approaches this maximum, the voltage required to draw the current would become unphysically large, and hence it is not possible to draw current in excess of this maximum.

\subsubsection{The potential drop across the cell in galvanostatic discharge}

We consider the particular case of a fully charged well-balanced battery and examine how the voltage varies under galvanostatic discharge. For the battery to be well balanced requires that both negative and positive electrode reactions should be exhausted when the battery is fully discharged, that is $c_{\mathrm{a}}=0$ and $c_{\mathrm{c}}=\mathcal{C}_{\mathrm{c}}$ at full discharge (see (3.8c)-(3.8d)). The quantities $c_{\mathrm{a}}$ and $c_{\mathrm{c}}$ have been non-dimensionalised with a typical intercalated lithium concentration $c_{\mathrm{s}}^{0}$ which we are free to choose. We make this choice so that $\mathcal{C}_{\mathrm{c}}=1$, and the initial conditions based on (4.19)-(4.20), at the beginning of the battery discharge, for the battery to be well balanced are then

$\left.c_{\mathrm{a}, 0}\right|_{t=0}=\frac{A}{B}(\Lambda-1)$ and $\left.c_{\mathrm{c}, 0}\right|_{t=0}=0$ where $A=\frac{\left|\hat{V}_{\mathrm{a}}\right|+\left|\hat{\Omega}_{\mathrm{a}}\right|}{\left|\hat{\Omega}_{\mathrm{a}}\right|}$ and $B=\frac{\left|\hat{V}_{\mathrm{c}}\right|+\left|\hat{\Omega}_{\mathrm{c}}\right|}{\left|\hat{\Omega}_{\mathrm{c}}\right|}$. 
In addition, it is required that $0<c_{\mathrm{a}, 0}<\mathcal{C}_{\mathrm{a}}$ and $0<c_{\mathrm{c}, 0}<1$ in order for the discharge to proceed. These models depend on the facts that both electrodes have a limited capacity for lithium and lithium concentration in the electrodes cannot go negative (see (3.8c)-(3.8d)).

The potential drop across the cell is given by

$\Phi_{0}(t)=U\left(c_{\mathrm{a}, 0}(t)\right)-V\left(c_{\mathrm{c}, 0}(t)\right)$.

The solution to this system is, where $I$ (and hence by (4.22) $\mu$ also) is constant,

$c_{\mathrm{a}, 0}=\aleph_{\mathrm{a}}=\frac{A}{B}(\Lambda-1)\left(1-\mathcal{D}_{\mathrm{d}}\right)$ in $0<x<1$,

$c_{\mathrm{c}, 0}=\aleph_{\mathrm{c}}=(\Lambda-1) \mathcal{D}_{\mathrm{d}}$ in $1<x<\mu$,

where the degree of discharge $\mathcal{D}_{\mathrm{d}}$ is defined by

$\mathcal{D}_{\mathrm{d}}=\frac{B}{\Lambda-1} \int_{0}^{t} I \mathrm{~d} t$

Notably the discharge finishes when $c_{\mathrm{c}, 0}=1$ when $\mathcal{D}_{\mathrm{d}}=(\mu-1) /(\Lambda-1)$. Defining the functions $\hat{U}$ and $\hat{V}^{-}$by $\hat{U}\left(\mathcal{D}_{\mathrm{d}}\right)=U\left(\frac{A}{B}(\Lambda-1)\left(1-\mathcal{D}_{\mathrm{d}}\right)\right)$ and $\hat{V}^{-}\left(\mathcal{D}_{\mathrm{d}}\right)=-V\left(\mathcal{D}_{\mathrm{d}}\right)$ for $0<\mathcal{D}_{\mathrm{d}}<1$,

leads to the following formulae for the discharge potential (derived from (4.24)) as a function of degree of discharge $\mathcal{D}_{\mathrm{d}}$ :

$\Phi_{0}\left(\mathcal{D}_{\mathrm{d}}\right)=\left\{\begin{array}{l}\hat{U}\left(\mathcal{D}_{\mathrm{d}}\right)+\hat{V}^{-}\left(\mathcal{D}_{\mathrm{d}}\right) \text { for } I<I_{\text {crit }} \text { and } 0<\mathcal{D}_{\mathrm{d}}<1 \\ \hat{U}\left(\mathcal{D}_{\mathrm{d}}\right)+\hat{V}^{-}\left(\frac{\Lambda-1}{\mu-1} \mathcal{D}_{d}\right) \text { for } I_{\text {crit }}<I<I_{\max } \text { and } 0<\mathcal{D}_{\mathrm{d}}<(\mu-1) /(\Lambda-1)\end{array}\right.$

Note that for the case $I<I_{\text {crit }}$ the leading order potential drop $\Phi_{0}$ is simply the OCV. However, in the large current case, the resulting voltage depends on characteristics of both the positive and negative electrodes in a non-standard fashion.

\subsubsection{Fraction of the utilised stored energy at high currents}

One of the characteristics of interest for a battery is the fraction of the stored energy that is utilised for a particular rate of galvanostatic discharge. To lowest order, because the battery is separated into a region of constant discharge rate and a region of no discharge by the free boundary, this fraction of utilised stored energy, which we denote by $\Theta$, can be determined by finding the proportion of the positive electrode $(\mu(I)-1) /(\Lambda-1)$ which participates in the discharge.

Assuming that the battery is well balanced as described in Sect. 4.2.2, then $\Theta$, as a function of the (steady) current $I$, is given by

$\Theta(I)=\frac{\mu(I)-1}{\Lambda-1}=\frac{1}{2(\Lambda-1)}\left(\left(9+\frac{48 \mathcal{B}_{+} \gamma \kappa_{\mathrm{p}} \Lambda}{I}-8 \frac{\mathcal{B}_{+}}{\mathcal{B}_{-}}\right)^{1 / 2}-3\right)$.

This can be re-expressed in terms of the critical and maximum currents as follows:

$\Theta(I)=\left(\frac{C^{2}-\Delta(C+1)^{2}+(2 C+1) I_{\text {crit }} / I}{1-\Delta}\right)^{1 / 2}-C$ where $C=\frac{3}{2(\Lambda-1)}$ and $\Delta=\frac{I_{\text {crit }}}{I_{\max }}$.

Plots of typical fractions of the utilised stored energy are shown in Fig. 7. These show that, at the small discharge rates $I<I_{\text {crit }}$, all the battery is used $\Theta=1$, and that above the critical current, the fraction of utilised stored energy drops until at the maximum current when none of the stored energy is used. 

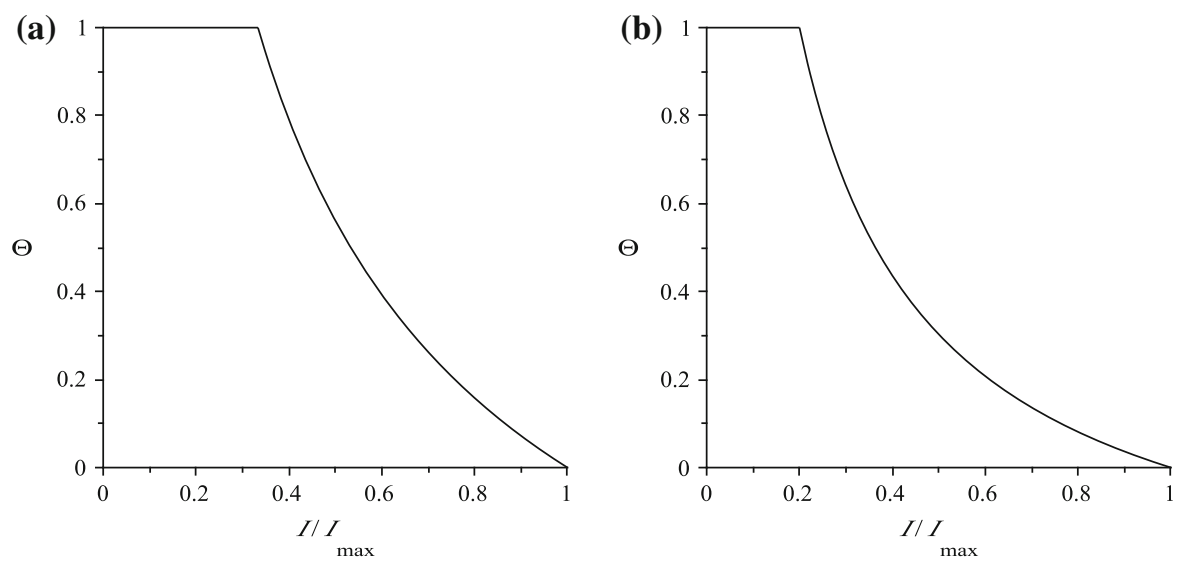

Fig. 7 Plots of $\Theta$ the fraction of the utilised stored energy, as a function of the galvanostatic discharge current $I$, for a $\Lambda=2$ (i.e. $C=3 / 2$ ) and $I_{\text {crit }} / I_{\max }=1 / 3$ (i.e. $\Delta=1 / 3$ ) and for $\mathbf{b} \Lambda=2$ and $I_{\text {crit }} / I_{\max }=1 / 5$

\subsection{Results of the large $\lambda$ asymptotics}

To illustrate the behaviour predicted by the simplified homogenised model, we consider a particular battery configuration with $\Lambda=2$ so that the positive electrode and negative electrode are of the same size. The distribution of the ions in the electrolyte is then determined using the analysis of Sect. 4. Various positive currents (corresponding to battery discharge) have been considered, and Figs. 4 and 5 show how the ion concentration changes in these different cases. Figure 4 a shows scenarios in which the current is small enough so that the reactions are significant everywhere while Fig. $4 \mathrm{~b}$ depicts scenarios in which the current is large enough so that the reaction almost ceases on the right-hand part of the positive electrode. In Fig. 5, similar results are given but here the diffusivity in the negative electrode is greater than that in the positive one due to, for example, having a looser packing of particles in the negative electrode. Consequently, the current required to create regions of ion depletion gets larger as the electrolyte effective diffusivity increases the positive electrode. The behaviour of the analytic solution for the ion concentrations, at currents $I>I_{\text {crit }}$, in Figs. $4 \mathrm{~b}$ and $5 \mathrm{~b}$ are qualitatively very similar to the numerical solutions presented by Doyle and Newman [5] and Fuller et al. [7] on the basis of a solution to the full model.

\section{Conclusion}

We formulated a detailed model of a lithium-ion battery accounting for the electrolyte, the electrode particles and the reactions at the interface between electrode particles and the electrolyte. Exploiting the small size of the particles used in the electrodes, we then used the method of multiple scales to homogenise this model over the microstructure in the case where diffusion of intercalated lithium within individual electrode particles occurs over a much faster timescale than that of lithium ions across the whole cell. Finally, we used asymptotic methods, based on parameters estimated from the literature, to attain a greatly simplified version of the homogenised model, which is applicable when open circuit potential curves of the electrode materials show appreciable changes with lithium concentration within the material. Thus, this approach turns out to be applicable to materials such as $\mathrm{Li}_{x} \mathrm{C}_{6}$ but not to materials such as $\mathrm{LiFePO}_{4}$ and $\mathrm{Li}_{y} \mathrm{Mn}_{2} \mathrm{O}_{4}$ which have extremely flat sections to their open circuit potentials. The asymptotic description of discharge from such materials remains an open problem that we shall investigate in a subsequent study.

The parameter estimates that we took from the literature leads us to arrive at a simplification of the homogeneous model in which most of the potential drop across the battery takes place across the Debye layers surrounding each electrode particle. Hence, battery potential closely tracks the OCV. The transport of lithium ions in the electrolyte only significantly affects cell performance if the current is sufficiently large that there is more or less complete 
depletion of lithium ions in some region of the electrolyte within the electrode. At these high loads, this process leads to non-reacting regions in an electrode which consequently places a higher load on the reacting part of the electrode causing it to deplete more rapidly. Indeed, transport in the electrolyte creates an upper limit on the magnitude of the current which may be drawn from a particular battery. Given that the battery operates away from these extreme situations, this then leads to the lowest order, the potential drop across the cell is the OCV and the electrodes are uniformly charged and discharged. There are small corrections to the zero current voltage (which we have calculated) and to the distribution of the lithium in electrodes, which depend on the current being drawn from the cell. We emphasise, however, that the asymptotics leading to this simplification of the homogenised model does not apply to electrodes composed of materials with extremely flat discharge curves (e.g. $\left.\mathrm{LiFePO}_{4}\right)$ and that to study these, it is necessary to investigate a different asymptotic regime to the one studied here.

We would like to emphasise that the homogenisation method employed in this study gives a systematic method for investigating the effects due to changes in electrode particle size and geometry on the behaviour of the battery. It can thus be applied to assess use of different particles in various applications. There is considerable scope to investigate the effects due to such changes on battery performance by determining the coefficients in the homogenised model using the method described in Appendix A. Other obvious extensions to this study can include the effects of electrical resistance within the electrode material and investigation of the effect of lithium diffusion within (large) electrode particles for which the timescale for solid-state lithium diffusion, within the electrode particles, is comparable to that for lithium-ion diffusion, through the electrolyte.

Finally, we point out that there is scope to extend the homogenisation method used here to three-scale battery problems such as those investigated by Dargaville and Farrell [3]. Here, graphite-coated agglomerations of electrode particles (termed crystals in [3]) are pressed together to form the electrode. There are thus three distinct scales provided by individual electrode particles (crystals), agglomerations and the entire electrode.

Acknowledgment The authors would like to thank Dr. Troy Farrell for a number of enlightening conversations and the referees for their helpful comments.

\section{Appendix A: Derivation of the homogenised diffusion equation with sources}

In this Appendix, we consider how we can derive homogenised equations, over the lengthscale of the electrode, for the processes occurring in the electrolyte where the electrolyte has a microstructure. This will be accomplished via the method of multiple scales (see e.g. [33-35]). In this instance, we take the lengthscale of the microstructure to be of $O(\epsilon)$ and that of the electrode to be of $O(1)$ where $\epsilon \ll 1$. Furthermore, we assume that the microstructure is locally periodic inside a completely periodic array of boxes which we denote by $\hat{V}_{\text {per }} \cup \hat{\Omega}_{\text {per }}$. Here, $\hat{V}_{\text {per }}$ represents the space inside this box occupied by the electrolyte while $\hat{\Omega}_{\text {per }}$ represents the space occupied by the electrode particles (see Fig. 3 as an illustrative configuration). For the sake of generality, we allow the microstructure to change slowly, over the $O(1)$ lengthscale, and it is in this sense that it is locally periodic.

Consider the following dimensionless equations and boundary conditions for $c$ and $\phi$

$$
\begin{aligned}
& \frac{\partial c}{\partial t}+\nabla \cdot \boldsymbol{q}=0 \text { where } \boldsymbol{q}=-D \nabla c \text { in } \hat{V}_{\mathrm{per}}, \\
& \left.\boldsymbol{q} \cdot \boldsymbol{n}\right|_{\partial \hat{\Omega}_{\mathrm{per}}}=\epsilon k Q, \\
& \nabla \cdot \boldsymbol{f}=0 \text { where } \boldsymbol{f}=-D(\lambda c \nabla \phi-\alpha \nabla c) \text { in } \hat{V}_{\mathrm{per}}, \\
& \left.\boldsymbol{f} \cdot \boldsymbol{n}\right|_{\partial \hat{\Omega}_{\mathrm{per}}}=\epsilon k(1-\alpha) Q,
\end{aligned}
$$

Here, $\boldsymbol{n}$ is the unit outward normal to $\hat{\Omega}_{\text {per }}$ and $\partial \hat{\Omega}_{\text {per }}$ the boundary to $\hat{\Omega}_{\text {per }}$ (see Fig. 3), and hence the unit inward normal to $\hat{V}_{\text {per }}$.

Multiple scales formulation. We investigate the distinguished limit that $k, D$ and $Q$ are $O(1)$ while $\epsilon \ll 1$ by introducing the microscale variable $\hat{\boldsymbol{x}}$ defined by

$\boldsymbol{x}=\epsilon \hat{\boldsymbol{x}}$. 
In terms of this new variable, the multiple scales expansion transforms the gradient operator via $\nabla \rightarrow \nabla+\hat{\nabla} / \epsilon$. We can thus rewrite (7.1)-(7.4) in the form:

$\frac{\partial c}{\partial t}+\frac{1}{\epsilon} \hat{\nabla} \cdot \boldsymbol{q}+\nabla \cdot \boldsymbol{q}=0, \quad \boldsymbol{q}=-D\left(\frac{1}{\epsilon} \hat{\nabla} c+\nabla c\right) \quad$ in $\hat{V}_{\mathrm{per}}$,

$\left.\boldsymbol{q} \cdot \boldsymbol{n}\right|_{\partial \hat{\Omega}_{\mathrm{per}}}=\epsilon k Q, \quad c$ and $\boldsymbol{q}$ periodic in $\hat{\boldsymbol{x}}$ on $\hat{V}_{\mathrm{per}}$,

$\frac{1}{\epsilon} \hat{\nabla} \cdot \boldsymbol{f}+\nabla \cdot \boldsymbol{f}=0, \quad \boldsymbol{f}=-D\left[\left(\frac{1}{\epsilon} \lambda c \hat{\nabla} \phi+\lambda c \nabla \phi\right)-\alpha\left(\frac{1}{\epsilon} \hat{\nabla} c+\nabla c\right)\right] \quad$ in $\hat{V}_{\mathrm{per}}$,

$\left.\boldsymbol{f} \cdot \boldsymbol{n}\right|_{\partial \hat{\Omega}_{\mathrm{per}}}=\epsilon k Q(1-\alpha), \quad \phi$ periodic in $\hat{\boldsymbol{x}}$ on $\hat{V}_{\mathrm{per}}$.

Here, we make the standard assumption that variables are periodic in the shortscale variable $\hat{\boldsymbol{x}}$. This ensures that there are no secular growth terms in their asymptotic expansions (in powers of $\epsilon$ ).

Mathematical preliminary. Before proceeding with the expansion, we re-emphasise that although the interface $\partial \hat{\Omega}_{\text {per }}$ is approximately periodic on the short lengthscale significant variations may occur over the longer $x$ lengthscale. We will find it useful to define this interface by the zero level set of the function $\psi$, that is by the relation $\psi(\hat{\boldsymbol{x}}, \boldsymbol{x})=0$. Furthermore, we define $\psi$ so that $\boldsymbol{n}$, the outward unit normal to $\hat{\Omega}_{\mathrm{per}}$, (or equivalently the unit inward normal to $\hat{V}_{\text {per }}$ ) is given by

$\boldsymbol{n}=-\frac{\hat{\nabla} \psi+\epsilon \nabla \psi}{|\hat{\nabla} \psi+\epsilon \nabla \psi|}$.

We are then able to calculate the rate of change of a quantity $A(\hat{\boldsymbol{x}}, \boldsymbol{x})$ integrated over the locally periodic domain $\hat{V}_{\text {per }}$ in terms of the surface function $\psi$ (see Sect. 7.1) finding it to be

$\frac{\partial}{\partial x_{i}} \int_{\hat{V}_{\mathrm{per}}} A(\hat{\boldsymbol{x}}, \boldsymbol{x}) \mathrm{d} \hat{V} \sim \int_{\hat{V}_{\mathrm{per}}} \frac{\partial A}{\partial x_{i}} \mathrm{~d} \hat{V}-\int_{\partial \hat{\Omega}_{\mathrm{per}}} A \frac{1}{|\hat{\nabla} \psi|} \frac{\partial \psi}{\partial x_{i}} \mathrm{~d} \hat{S}$.

In turn, we can use the above result to show that

$\nabla \cdot \int_{\hat{V}_{\mathrm{per}}} \boldsymbol{q}(\hat{\boldsymbol{x}}, \boldsymbol{x}) \mathrm{d} \hat{V} \sim \int_{\hat{V}_{\mathrm{per}}} \nabla \cdot \boldsymbol{q} \mathrm{d} \hat{V}-\int_{\partial \hat{\Omega}_{\mathrm{per}}} \frac{\boldsymbol{q} \cdot \nabla \psi}{|\hat{\nabla} \psi|} \mathrm{d} \hat{S}$.

The asymptotic expansion. We now expand the variables in (7.5)-(7.8) as follows:

$c=c_{0}(\boldsymbol{x}, t)+\epsilon c_{1}(\hat{\boldsymbol{x}}, \boldsymbol{x}, t)+\epsilon^{2} c_{2}(\hat{\boldsymbol{x}}, \boldsymbol{x} t)+\ldots$,

$\phi=\frac{1}{\lambda}\left(\tilde{\phi}_{0}(\boldsymbol{x}, t)+\epsilon \tilde{\phi}_{1}(\hat{\boldsymbol{x}}, \boldsymbol{x}, t)+\epsilon^{2} \tilde{\phi}_{2}(\hat{\boldsymbol{x}}, \boldsymbol{x} t)+\ldots\right)$,

$\boldsymbol{q}=\boldsymbol{q}_{0}(\hat{\boldsymbol{x}}, \boldsymbol{x}, t)+\epsilon \boldsymbol{q}_{1}(\hat{\boldsymbol{x}}, \boldsymbol{x}, t)+\epsilon^{2} \boldsymbol{q}_{2}(\hat{\boldsymbol{x}}, \boldsymbol{x}, t)+\ldots$,

$\boldsymbol{f}=\boldsymbol{f}_{0}(\hat{\boldsymbol{x}}, \boldsymbol{x}, t)+\epsilon \boldsymbol{f}_{1}(\hat{\boldsymbol{x}}, \boldsymbol{x}, t)+\epsilon^{2} \boldsymbol{f}_{2}(\hat{\boldsymbol{x}}, \boldsymbol{x}, t)+\ldots$

Derivation of a solvability condition on $c_{0}$. Consider first the flux equations for $\boldsymbol{q}$ (7.5)-(7.6). To leading order, we obtain

$\hat{\nabla} \cdot \boldsymbol{q}_{0}=0,\left.\quad \boldsymbol{q}_{0} \cdot \boldsymbol{n}\right|_{\partial \hat{\Omega}_{\mathrm{per}}}=0, \quad \boldsymbol{q}_{0}$ periodic in $\hat{\boldsymbol{x}}$ on $\hat{V}_{\mathrm{per}}$.

At $O(\epsilon)$ in (7.5)-(7.6) we find

$\frac{\partial c_{0}}{\partial t}+\hat{\nabla} \cdot \boldsymbol{q}_{1}+\nabla \cdot \boldsymbol{q}_{0}=0, \quad \boldsymbol{q}_{1}$ periodic in $\hat{\boldsymbol{x}}$ on $\hat{V}_{\mathrm{per}}$,

$\left.\boldsymbol{q}_{1} \cdot \boldsymbol{n}\right|_{\partial \hat{\Omega}_{\mathrm{per}}}=k Q$.

Rewriting the boundary condition (7.13) in terms of the surface function $\psi(\hat{\boldsymbol{x}}, \boldsymbol{x})$ (recalling that the normal to $\partial \Omega$ is given by (7.9)) gives

$\boldsymbol{q}_{1} \cdot \hat{\nabla} \psi+\left.\boldsymbol{q}_{0} \cdot \nabla \psi\right|_{\partial \hat{\Omega}_{\mathrm{per}}}=-|\hat{\nabla} \psi| k Q$. 
Integrating (7.12) over $\hat{V}_{\text {per }}$ and using the fact the outward normal to $\hat{V}_{\text {per }}$ on that section of the boundary it shares with $\hat{\Omega}_{\text {per }}$ is, to leading order in $\epsilon, \hat{\nabla} \psi /|\hat{\nabla} \psi|$ results in the integral equations:

$\int_{\hat{V}_{\text {per }}} \frac{\partial c_{0}}{\partial t}+\nabla \cdot \boldsymbol{q}_{0} \mathrm{~d} \hat{V}+\int_{\partial \hat{\Omega}_{\mathrm{per}}} \boldsymbol{q}_{1} \cdot \frac{\hat{\nabla} \psi}{|\hat{\nabla} \psi|} \mathrm{d} \hat{S}=0$,

which in turn, on substitution of (7.14) gives

$\int_{\hat{V}_{\mathrm{per}}} \frac{\partial c_{0}}{\partial t}+\nabla \cdot \boldsymbol{q}_{0} \mathrm{~d} \hat{V}-\int_{\partial \hat{\Omega}_{\mathrm{per}}} \boldsymbol{q}_{0} \cdot \frac{\nabla \psi}{|\hat{\nabla} \psi|} \mathrm{d} \hat{S}=\int_{\partial \hat{\Omega}_{\mathrm{per}}} k Q \mathrm{~d} \hat{S}$,

Finally, we use the result (7.10) to rewrite this in the following preferred form

$\int_{\hat{V}_{\text {per }}} \frac{\partial c_{0}}{\partial t} \mathrm{~d} \hat{V}+\nabla \cdot \int_{\hat{V}_{\text {per }}} \boldsymbol{q}_{0} \mathrm{~d} \hat{V}=\int_{\partial \hat{\Omega}_{\text {per }}} k Q \mathrm{~d} \hat{S}$.

It remains to determine $\boldsymbol{q}_{0}$ in terms of $c_{0}$. To leading order in $(7.5 \mathrm{~b})$, we find

$\boldsymbol{q}_{0}=-D\left(\nabla c_{0}+\hat{\nabla} c_{1}\right)$.

Substitution of this into (7.11) yields

$\hat{\nabla}^{2} c_{1}=0,\left.\quad \boldsymbol{n} \cdot \hat{\nabla} c_{1}\right|_{\partial \hat{\Omega}_{\mathrm{per}}}=-\left.\boldsymbol{n} \cdot \nabla c_{0}\right|_{\partial \hat{\Omega}_{\mathrm{per}}}, \quad c_{1}$ periodic in $\hat{\boldsymbol{x}}$ on $\hat{V}_{\text {per }}$.

We make use of the linearity of this problem to write its solution in the form:

$c_{1}=-\left(\frac{\partial c_{0}}{\partial x_{1}} \chi^{(1)}(\hat{\boldsymbol{x}}, \boldsymbol{x})+\frac{\partial c_{0}}{\partial x_{2}} \chi^{(2)}(\hat{\boldsymbol{x}}, \boldsymbol{x})+\frac{\partial c_{0}}{\partial x_{3}} \chi^{(3)}(\hat{\boldsymbol{x}}, \boldsymbol{x})\right)$,

where the basis functions $\chi^{(i)}$ satisfy the following problems:

$\hat{\nabla}^{2} \chi^{(i)}=0$ in $\hat{V}_{\text {per }}$,

$\left.\hat{\nabla} \chi^{(i)} \cdot \boldsymbol{n}\right|_{\partial \hat{\Omega}_{\mathrm{per}}}=\left.\boldsymbol{e}_{i} \cdot \boldsymbol{n}\right|_{\partial \hat{\Omega}_{\mathrm{per}}}$,

$\chi^{(i)}$ periodic in $\hat{\boldsymbol{x}}$ on $\hat{V}_{\text {per }}$,

for $i=1,2,3$

$\int_{\hat{V}_{\text {per }}} \chi^{(i)} \mathrm{d} \hat{V}=0$

and $\boldsymbol{e}_{i}$ is a basis vector in the $x_{i}$-direction. Here, the final integral condition is imposed, which ensures uniqueness of the solution but does not change any of the results we derive, since these all depend on the derivatives of $\chi^{(i)}$ rather than $\chi^{(i)}$ itself. It follows that the leading order flux is given by

$\boldsymbol{q}_{0}=-D\left(\delta_{i j}-\frac{\partial \chi^{(j)}}{\partial \hat{x}_{i}}\right) \frac{\partial c_{0}}{\partial x_{j}} \boldsymbol{e}_{i}$.

where the Einstein summation convention is used. Substitution of this result so obtained into (7.15) then yields an equation for $c_{0}$ in terms of the macroscopic variable $\boldsymbol{x}$

$\frac{\left|\hat{V}_{\mathrm{per}}\right|}{\left|\hat{V}_{\mathrm{per}}\right|+\left|\hat{\Omega}_{\mathrm{per}}\right|} \frac{\partial c_{0}}{\partial t}=D \frac{\partial}{\partial x_{i}}\left(\mathcal{B}_{i j} \frac{\partial c_{0}}{\partial x_{j}}\right)+k \tilde{Q}$,

where

$\left|\hat{V}_{\text {per }}\right|=\int_{\hat{V}_{\text {per }}} \mathrm{d} \hat{V}, \quad\left|\hat{\Omega}_{\text {per }}\right|=\int_{\hat{\Omega}_{\text {per }}} \mathrm{d} \hat{V}, \quad \mathcal{B}_{i j}=\frac{\int_{\hat{V}_{\text {per }}} \delta_{i j}-\frac{\partial \chi^{(j)}}{\partial \hat{x}_{i}} \mathrm{~d} \hat{V}}{\left|\hat{V}_{\text {per }}\right|+\left|\hat{\Omega}_{\text {per }}\right|}, \quad \tilde{Q}=\frac{\int_{\partial \hat{\Omega}_{\text {per }}} Q \mathrm{~d} \hat{S}}{\left|\hat{V}_{\text {per }}\right|+\left|\hat{\Omega}_{\text {per }}\right|}$, 
and we have used the fact that $\left|\hat{\Omega}_{\text {per }}\right|+\left|\hat{V}_{\text {per }}\right|$, the dimensionless volume of the cell, is constant throughout the domain.

Derivation of a solvability condition on $\tilde{\phi}_{0}$. The derivation of the solvability condition for $\tilde{\phi}_{0}$ proceeds along very similar lines to that for $c_{0}$. At leading order in the flux equations for $f$, namely (7.7), we find

$\hat{\nabla} \cdot \boldsymbol{f}_{0}=0,\left.\quad \boldsymbol{f}_{0} \cdot \boldsymbol{n}\right|_{\partial \hat{\Omega}_{\mathrm{per}}}=0, \quad \boldsymbol{f}_{0}$ periodic in $\hat{\boldsymbol{x}}$ on $\hat{V}_{\text {per }}$.

At next order, we find an equation for $\boldsymbol{f}_{1}$ which we can integrate in a similar manner to that for $\boldsymbol{q}_{1}$ to obtain the following solvability condition on $f_{0}$ :

$\nabla \cdot \int_{\hat{V}_{\text {per }}} f_{0} \mathrm{~d} \hat{V}=k(1-\alpha) \int_{\partial \hat{\Omega}_{\mathrm{per}}} Q \mathrm{~d} \hat{S}$.

Substituting the expansions for $c$ and $\phi$ into (7.7b) gives the following expression for $\boldsymbol{f}_{0}$ :

$\boldsymbol{f}_{0}=-D\left(c_{0} \hat{\nabla} \tilde{\phi}_{1}-\alpha \hat{\nabla} c_{1}+c_{0} \nabla \tilde{\phi}_{0}-\alpha \nabla c_{0}\right)$.

In turn, substitution of this expression into (7.20) yields a system for $\tilde{\phi}_{1}$

$\hat{\nabla}^{2} \tilde{\phi}_{1}=0,\left.\quad \hat{\nabla} \tilde{\phi}_{1} \cdot \boldsymbol{n}\right|_{\partial \hat{\Omega}_{\mathrm{per}}}=-\left.\nabla \tilde{\phi}_{0} \cdot \boldsymbol{n}\right|_{\partial \hat{\Omega}_{\mathrm{per}}}$,

with solution

$\tilde{\phi}_{1}=-\left(\frac{\partial \tilde{\phi}_{0}}{\partial x_{1}} \chi^{(1)}(\hat{\boldsymbol{x}}, \boldsymbol{x})+\frac{\partial \tilde{\phi}_{0}}{\partial x_{2}} \chi^{(2)}(\hat{\boldsymbol{x}}, \boldsymbol{x})+\frac{\partial \tilde{\phi}_{0}}{\partial x_{3}} \chi^{(3)}(\hat{\boldsymbol{x}}, \boldsymbol{x})\right)$,

from which it follows that

$\boldsymbol{f}_{0}=-D\left(\delta_{i j}-\frac{\partial \chi^{(j)}}{\partial \hat{x}_{i}}\right)\left(c_{0} \frac{\partial \tilde{\phi}_{0}}{\partial x_{j}}-\alpha \frac{\partial c_{0}}{\partial x_{j}}\right) \boldsymbol{e}_{i}$.

On substitution of this expression into (7.21), we obtain an equation for $\tilde{\phi}_{0}$

$D \frac{\partial}{\partial x_{i}}\left(\mathcal{B}_{i j}\left(c_{0} \frac{\partial \tilde{\phi}_{0}}{\partial x_{j}}-\alpha \frac{\partial c_{0}}{\partial x_{j}}\right)\right)+k(1-\alpha) \tilde{Q}=0$.

In summary, in (7.18) and (7.23), we have derived macroscale equations for the leading order behaviour of $c$ and $\phi$ which, where we recall that $\phi \sim \tilde{\phi}_{0} / \lambda$ and drop subscripts, can be written in the form:

$\frac{\left|\hat{V}_{\text {per }}\right|}{\left|\hat{V}_{\text {per }}\right|+\left|\hat{\Omega}_{\text {per }}\right|} \frac{\partial c}{\partial t}=D \frac{\partial}{\partial x_{i}}\left(\mathcal{B}_{i j} \frac{\partial c}{\partial x_{j}}\right)+k \tilde{Q}$

$D \frac{\partial}{\partial x_{i}}\left(\mathcal{B}_{i j}\left(\lambda c \frac{\partial \phi}{\partial x_{j}}-\alpha \frac{\partial c}{\partial x_{j}}\right)\right)+k(1-\alpha) \tilde{Q}=0$.

Notably these equations are both in conservation form.

Appendix A.1: Accounting for $O(1)$ lengthscale variations of the microscopic geometry

Consider the surface $\partial \hat{\Omega}_{\text {per }}$ defined by $\psi(\hat{\boldsymbol{x}}, \boldsymbol{x})=0$. Alternatively, we can write this in the form: $\hat{\boldsymbol{x}}=\boldsymbol{r}\left(\tau_{1}, \tau_{2}, \boldsymbol{x}\right)$ so that $\psi(\boldsymbol{r}, \boldsymbol{x})=0$. Since $\psi\left(\boldsymbol{r}+\mathrm{d} \boldsymbol{r}, \boldsymbol{x}+\mathrm{d} x_{i} \boldsymbol{e}_{i}\right)=0$ it follows that

$\frac{\partial \boldsymbol{r}}{\partial x_{i}} \cdot \hat{\nabla} \psi=-\frac{\partial \psi}{\partial x_{i}}$.

Because the unit inward normal to $\hat{V}_{\text {per }}$ is defined, in terms of $\psi$, by $\boldsymbol{n}=-\hat{\nabla} \psi /|\hat{\nabla} \psi|+O(\epsilon)$, we can rewrite the above in the form:

$\frac{\partial \boldsymbol{r}}{\partial x_{i}} \cdot \boldsymbol{n}=\frac{1}{|\hat{\nabla} \psi|} \frac{\partial \psi}{\partial x_{i}}+O(\epsilon)$ 
Fig. 8 Illustration of the change in the electrode boundary shape (viewed as a function of the shortscale variable $\hat{\boldsymbol{x}}$ ) with a small displacement $d \boldsymbol{x}$ of the longscale variable $\boldsymbol{x}$

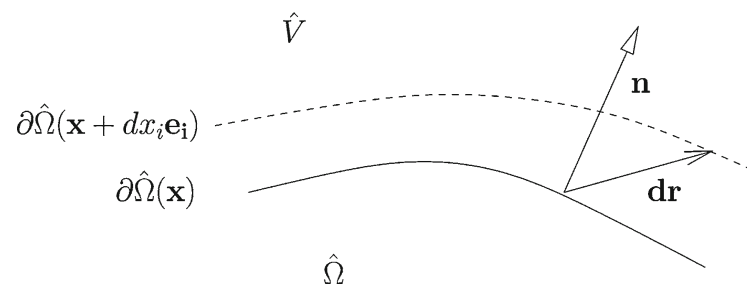

$\hat{\Omega}$

(see Fig. 8 for an illustration on this). We can identify the quantity $\mathrm{d} x_{i} \frac{\partial \boldsymbol{r}}{\partial x_{i}} \cdot \boldsymbol{n}$ as the normal distance between the surface $\partial \hat{\Omega}_{\text {per }}$ at $\boldsymbol{x}$ and $\partial \hat{\Omega}_{\text {per }}$ at $\boldsymbol{x}+\mathrm{d} x_{i} \boldsymbol{e}_{i}$. The rate of change of $\int_{\hat{V}_{\text {per }}} A(\hat{\boldsymbol{x}}, \boldsymbol{x}) \mathrm{d} \hat{V}$ is thus composed of two terms, the first representing the rate of change of $A$ with $x_{i}$ and the second arising from the rate of change of the boundary to $V$ with $x_{i}$ which can be written as the surface integral

$-\int_{\partial \hat{\Omega}_{\mathrm{per}}} A \frac{\partial \boldsymbol{r}}{\partial x_{i}} \cdot \boldsymbol{n} \mathrm{d} \hat{S}$

It follows that

$\frac{\partial}{\partial x_{i}} \int_{\hat{V}_{\mathrm{per}}} A(\hat{\boldsymbol{x}}, \boldsymbol{x}) \mathrm{d} \hat{V} \sim \int_{\hat{V}_{\mathrm{per}}} \frac{\partial A}{\partial x_{i}} \mathrm{~d} \hat{V}-\int_{\partial \hat{\Omega}_{\mathrm{per}}} A \frac{1}{|\hat{\nabla} \psi|} \frac{\partial \psi}{\partial x_{i}} \mathrm{~d} \hat{S}$.

Here, the first term on the right-hand side represents the change in $A$ with $x_{i}$, while the second term arises because the domain $\hat{V}_{\text {per }}$ changes.

Appendix A.2: Properties of the tensor $\mathcal{B}_{i j}$

There are a number of results which help in illustrating the behaviour of the tensor $\mathcal{B}_{i j}$. The most of important of these are

$$
\begin{aligned}
& \int_{\hat{V}_{\mathrm{per}}} \frac{\partial \chi^{(j)}}{\partial \hat{x}_{i}} \mathrm{~d} \hat{V}=-\int_{\partial \hat{\Omega}_{\mathrm{per}}} \chi^{(j)} \boldsymbol{e}_{i} \cdot \boldsymbol{n} \mathrm{d} \hat{S}, \\
& \int_{\hat{V}_{\mathrm{per}}} \frac{\partial \chi^{(j)}}{\partial \hat{x}_{i}} \mathrm{~d} \hat{V}=\int_{\hat{V}_{\mathrm{per}}} \frac{\partial \chi^{(i)}}{\partial \hat{x}_{j}} \mathrm{~d} \hat{V}, \\
& \int_{\hat{V}_{\mathrm{per}}} \frac{\partial \chi^{(i)}}{\partial \hat{x}_{i}} \mathrm{~d} \hat{V}>0 \quad \text { (no summation). }
\end{aligned}
$$

The first of these results can be demonstrated by a straightforward application of the divergence theorem; the proofs for the second and third result are given below.

We can use results (7.28)-(7.29) to show that

$\mathcal{B}_{i j}=\mathcal{B}_{j i}, \quad \mathcal{B}_{i i}<1 . \quad$ (no summation).

Thus, the tensor $\mathcal{B}_{i j}$ is symmetric and furthermore, the effective diffusivity in any particular direction is reduced by the microstructure below the one which would be observed without microstructure (which is precisely what one expects). In particular, for a uniform isotropic microstructure, we have (as previously stated) $\mathcal{B}_{i j}=\mathcal{B} \delta_{i j}$ with $\mathcal{B}>0$. It follows that the effective diffusivity $D \mathcal{B}$ is smaller than the diffusivity $D$ in the absence of microstructure.

We start by demonstrating that (7.28) is true before using this result to show that (7.29) holds. 
Proof of (7.28). Consider the integral $I$ defined by

$I=\int_{\hat{V}_{\mathrm{per}}} \hat{\nabla} \cdot\left(\chi^{(i)} \hat{\nabla} \chi^{(k)}-\chi^{(k)} \hat{\nabla} \chi^{(i)}\right) \mathrm{d} \hat{V}=\int_{\hat{V}_{\mathrm{per}}}\left(\chi^{(i)} \hat{\nabla}^{2} \chi^{(k)}-\chi^{(k)} \hat{\nabla}^{2} \chi^{(i)}\right) \mathrm{d} \hat{V}$

It follows from the definition of $\chi^{(i)}$ (see (7.17)) that $I=0$. If we now apply the divergence theorem to $I$, then we find that

$I=-\int_{\partial \hat{\Omega}_{\mathrm{per}}}\left(\chi^{(i)} \hat{\nabla} \chi^{(k)}-\chi^{(k)} \hat{\nabla} \chi^{(i)}\right) \cdot \boldsymbol{n} \mathrm{d} \hat{S}$.

Referring to the boundary conditions on $\chi^{(i)}$ on $\partial \hat{\Omega}_{\text {per }}$ contained in (7.17) and using the fact that $I=0$ leads to the result

$\int_{\partial \hat{\Omega}_{\mathrm{per}}} \chi^{(k)} \boldsymbol{e}_{i} \cdot \boldsymbol{n} \mathrm{d} \hat{S}=\int_{\partial \hat{\Omega}_{\mathrm{per}}} \chi^{(i)} \boldsymbol{e}_{k} \cdot \boldsymbol{n} \mathrm{d} \hat{S}$,

and hence by the result (7.27) to the desired result, namely (7.28).

Proof of (7.29). We will show that

$-\int_{\partial \hat{\Omega}_{\mathrm{per}}} \chi^{(i)} \boldsymbol{e}_{i} \cdot \boldsymbol{n} \mathrm{d} \hat{S}>0$ no summation over $i$,

and hence, by (7.27), that (7.29) is true. It follows that the trace of the diffusivity tensor is increased by the microstructure, as might be expected. In order to see this, consider multiplying (7.17) by $\chi^{(i)}$ to show that

$\hat{\nabla} \cdot\left(\chi^{(i)} \hat{\nabla} \chi^{(i)}\right)=\left|\hat{\nabla} \chi^{(i)}\right|^{2}$

$\left.\left.\chi^{(i)} \hat{\nabla} \chi^{(i)} \cdot \boldsymbol{n}\right|_{\partial \hat{\Omega}_{\mathrm{per}}}=\left.\chi^{(i)} \boldsymbol{e}_{i} \cdot \boldsymbol{n}\right|_{\partial \hat{\Omega}_{\mathrm{per}}}\right\} \quad$ for $i=1,2,3$ no summation over $i$.

$\chi^{(i)} \hat{\nabla} \chi^{(i)}$ periodic in $\hat{\boldsymbol{x}}$ on $\hat{V}_{\text {per }}$

Integrating the first of these equations over $V$ gives

$\int_{\hat{V}_{\mathrm{per}}}\left|\hat{\nabla} \chi^{(i)}\right|^{2} \mathrm{~d} \hat{V}=\int_{\partial \hat{V}_{\mathrm{per}} \backslash \partial \hat{\Omega}_{\mathrm{per}}} \chi^{(i)} \hat{\nabla} \chi^{(i)} \cdot \boldsymbol{n} \mathrm{d} \hat{S}-\int_{\partial \hat{\Omega}_{\mathrm{per}}} \chi^{(i)} \hat{\nabla} \chi^{(i)} \cdot \boldsymbol{n} \mathrm{d} \hat{S}$.

The first term on the right-hand side of this equations vanishes by periodicity. Applying the boundary condition on $\partial \hat{\Omega}_{\text {per }}$ contained in (7.32) leads to

$-\int_{\partial \hat{\Omega}_{\mathrm{per}}} \chi^{(i)} \boldsymbol{e}_{i} \cdot \boldsymbol{n} \mathrm{d} \hat{S}=\int_{\hat{V}_{\mathrm{per}}}\left|\hat{\nabla} \chi^{(i)}\right|^{2} \mathrm{~d} \hat{V}>0, \quad$ no summation over $i$

which is the result (7.31) from which (7.29) follows.

\section{Appendix B: Alternative forms for the Butler-Volmer condition}

An alternative form for these conditions is given, in the case of the negative electrode composed of $\mathrm{Li}_{1-x} \mathrm{CoO}_{2}$, by [13] while a generic version for any oxidative/reductive reaction involving a single species is given by [37, Chap. 12] and takes the form:

$$
G_{\mathrm{a}}^{*}=k_{1} c_{\mathrm{a}}^{*} \exp \left(-\left(1-\alpha_{\mathrm{a}}\right) \frac{F}{R_{\mathrm{g}} T}\left(\phi^{*}-U^{*}\left(c_{\mathrm{a}}^{*}\right)\right)\right)-\left.k_{2} c^{*}\left(c_{\mathrm{a}}^{\max }-c_{\mathrm{a}}^{*}\right) \exp \left(\alpha_{\mathrm{a}} \frac{F}{R_{\mathrm{g}} T}\left(\phi^{*}-U^{*}\left(c_{\mathrm{a}}^{*}\right)\right)\right)\right|_{\partial \Omega_{\mathrm{ae}}},
$$


where the constants $k_{1}$ and $k_{2}$ are reaction rates. In the case of the positive electrode, the analogous alternative form given by [37, Chap. 12] is

$G_{\mathrm{c}}^{*}=k_{3} c_{\mathrm{c}}^{*} \exp \left(-\left(1-\alpha_{\mathrm{c}}\right) \frac{F}{R_{\mathrm{g}} T}\left(\phi^{*}-\Phi^{*}-V^{*}\left(c_{\mathrm{c}}^{*}\right)\right)\right)$
$-\left.k_{4} c^{*}\left(c_{\mathrm{c}}^{\max }-c_{\mathrm{c}}^{*}\right) \exp \left(\alpha_{\mathrm{c}} \frac{F}{R_{\mathrm{g}} T}\left(\phi^{*}-\Phi^{*}-V^{*}\left(c_{\mathrm{c}}^{*}\right)\right)\right)\right|_{\partial \Omega_{\mathrm{ce}}}$.

In general, the Butler-Volmer condition can be expressed in the form:

$$
\begin{aligned}
G^{*}=\mathcal{K}\left(c^{*}, c_{s}^{*}\right)[ & \exp \left(-(1-\alpha) \frac{F}{R_{\mathrm{g}} T}\left(\phi^{*}-\phi_{\text {elec }}^{*}-\tilde{U}^{*}\left(c^{*}, c_{\mathrm{s}}^{*}\right)\right)\right) \\
& \left.-\exp \left(\alpha \frac{F}{R_{\mathrm{g}} T}\left(\phi^{*}-\phi_{\text {elec }}^{*}-\tilde{U}^{*}\left(c^{*}, c_{\mathrm{s}}^{*}\right)\right)\right)\right]\left.\right|_{\partial \Omega}
\end{aligned}
$$

where $\alpha$ is the transfer coefficient and $G^{*}, \phi_{\mathrm{elec}}^{*}$ and $c_{\mathrm{s}}^{*}$ are, respectively, the reaction rate, the potential, the intercalated lithium concentration on the surface of the electrode while $\mathcal{K}$ is a potential dependent pre-factor. Expressed in this form, it is clear that the potential drop between the electrolyte and the electrode $\phi^{*}-\phi_{\text {elec }}^{*}$ is, at equilibrium, $\tilde{U}^{*}\left(c^{*}, c_{\mathrm{S}}^{*}\right)$. In the cases of (2.6) and (2.8), this equilibrium drop is clearly given by $U^{*}\left(c_{\mathrm{a}}^{*}\right)$ and $V^{*}\left(c_{\mathrm{c}}^{*}\right)$, respectively, and is thus independent of the electrolyte concentration. However, in (B.1), the equilibrium drop is not given by $U\left(c_{\mathrm{a}}^{*}\right)$ but rather by $U^{*}\left(c_{\mathrm{a}}^{*}\right)-(R T / F) \log \left(k_{2} c^{*}\left(c_{\mathrm{a}}^{\max }-c_{\mathrm{a}}^{*}\right) /\left(k_{1} c_{\mathrm{a}}^{*}\right)\right)$ (which is $\tilde{U}^{*}\left(c^{*}, c_{\mathrm{a}}^{*}\right)$ in this case). Hence, we prefer the notation used in (2.6), (2.8) and (B.3) rather than that in (B.1) and (B.2). In the forthcoming analysis, we will exploit asymptotic methods and find that typically the potential drop between electrolyte and electrode in (B.1) and (B.2) is to leading order just $U^{*}\left(c_{\mathrm{a}}^{*}\right)$ and $V^{*}\left(c_{\mathrm{c}}^{*}\right)$, respectively: the corrections being only of the size of a thermal voltage, which is typically much smaller than the potential drop. Hence, we will find that, to leading order in the subsequent analysis, conditions (2.6) and (2.8) are equivalent to (B.1) and (B.2) but that the finer details are dependent on the exact conditions considered.

\section{Appendix C: The correction to the zero current voltage}

We now seek to determine $\Phi_{1}$ the next term in the expansion of the potential drop across the cell. In contrast to the leading term $\Phi_{0}$ (the zero current voltage), we expect this to depend directly on the current flowing in the circuit.

On elimination of the reaction terms appearing on the right-hand side of (3.8a) and (3.8b), we obtain the following:

$$
\frac{1}{\lambda} \frac{\left|\hat{V}_{\text {per }}\right|}{\gamma \tilde{\nu} \kappa_{\mathrm{n}}\left(\left|\hat{\Omega}_{\text {per }}\right|+\left|\hat{V}_{\text {per }}\right|\right)} \frac{\partial c}{\partial t}+\frac{\partial}{\partial x}\left(\left(\mathcal{B}_{-} H(x-1)+\mathcal{B}_{+} H(1-x)\right)\left(\lambda c \frac{\partial \phi}{\partial x}-\frac{\partial c}{\partial x}\right)\right)=0 .
$$

The leading term in this equation, on substituting the expansion (4.2), is

$$
\frac{\partial}{\partial x}\left(\left(\mathcal{B}_{-} H(x-1)+\mathcal{B}_{+} H(1-x)\right)\left(c_{0} \frac{\partial \phi_{1}}{\partial x}-\frac{\partial c_{0}}{\partial x}\right)\right)=0
$$

Boundary conditions on (C.2) are found from substituting (4.2) into (3.8i) from which we find that both $\phi_{1, x}$ and $c_{0, x}$ are zero on both $x=0$ and $x=\Lambda$. It follows that

$$
\left.\left(c_{0} \frac{\partial \phi_{1}}{\partial x}-\frac{\partial c_{0}}{\partial x}\right)\right|_{x=0}=0,\left.\quad\left(c_{0} \frac{\partial \phi_{1}}{\partial x}-\frac{\partial c_{0}}{\partial x}\right)\right|_{x=\Lambda}=0 .
$$

Integrating (C.2) and applying the boundary conditions gives the solution for $\phi_{1}$

$$
\phi_{1}=\log c_{0}(x)+F(t) \text {, }
$$


where $F(t)$ is a function of integration. In order to determine $F$ and $\Phi_{1}$, we proceed to first order in the Butler-Volmer conditions (3.8c)-(3.8d) substituting for $\tilde{G}_{\mathrm{a} 0}$ and $\tilde{G}_{\mathrm{c} 0}$ from (4.8) to find

$$
\begin{aligned}
& \tilde{M}_{1} c_{\mathrm{a}, 0}^{1 / 2}\left(\mathcal{C}_{\mathrm{a}}-c_{\mathrm{a}, 0}\right)^{1 / 2} c_{0}^{1 / 2}\left[\exp \left(\frac{1}{2}\left(c_{\mathrm{a}, 1} U^{\prime}\left(c_{\mathrm{a}, 0}\right)-\phi_{1}\right)\right)-\exp \left(\frac{1}{2}\left(\phi_{1}-c_{\mathrm{a}, 1} U^{\prime}\left(c_{\mathrm{a}, 0}\right)\right)\right)\right]=p I, \\
& \tilde{M}_{2} c_{\mathrm{c}, 0}^{1 / 2}\left(\mathcal{C}_{\mathrm{c}}-c_{\mathrm{c}, 0}\right)^{1 / 2} c_{0}^{1 / 2}\left[\exp \left(\frac{1}{2}\left(c_{\mathrm{c}, 1} V^{\prime}\left(c_{\mathrm{c}, 0}\right)+\Phi_{1}-\phi_{1}\right)\right)-\exp \left(\frac{1}{2}\left(\phi_{1}-\Phi_{1}-c_{\mathrm{c}, 1} V^{\prime}\left(c_{\mathrm{c}, 0}\right)\right)\right)\right]=-\frac{p I}{\Lambda-1},
\end{aligned}
$$

The expressions above are quadratic equations for the quantities $\mathcal{G}$ and $\mathcal{H}$ defined by

$2 \log \mathcal{G}(x, t)=c_{\mathrm{a}, 1} U^{\prime}\left(c_{\mathrm{a}, 0}\right)-\phi_{1}, \quad$ and $2 \log \mathcal{H}(x, t)=c_{\mathrm{c}, 1} V^{\prime}\left(c_{\mathrm{c}, 0}\right)+\Phi_{1}-\phi_{1}$,

with solutions

$$
\mathcal{G}=\frac{p I+\left(p^{2} I^{2}+4 \tilde{M}_{1}^{2} c_{0} c_{\mathrm{a}, 0}\left(\mathcal{C}_{\mathrm{a}}-c_{\mathrm{a}, 0}\right)\right)^{1 / 2}}{2 \tilde{M}_{1} c_{\mathrm{a}, 0}^{1 / 2} c_{0}^{1 / 2}\left(\mathcal{C}_{\mathrm{a}}-c_{\mathrm{a}, 0}\right)^{1 / 2}}, \quad \mathcal{H}=\frac{-p I+\left(p^{2} I^{2}+4(\Lambda-1)^{2} \tilde{M}_{2}^{2} c_{0} c_{\mathrm{c}, 0}\left(\mathcal{C}_{\mathrm{c}}-c_{\mathrm{c}, 0}\right)\right)^{1 / 2}}{2 \tilde{M}_{2}(\Lambda-1) c_{\mathrm{c}, 0}^{1 / 2} c_{0}^{1 / 2}\left(\mathcal{C}_{\mathrm{c}}-c_{\mathrm{c}, 0}\right)^{1 / 2}} .
$$

Note that in (C.5) $\mathcal{G}$ and $\mathcal{H}$ are expressed in terms of quantities that are determined as part of the leading order solution. Thus, we could determine $F(t)$ (the function of integration in (C.3)) and $\Phi_{1}(t)$ (the correction to the zero current voltage) by finding $c_{\mathrm{a}, 1}$ and $c_{\mathrm{c}, 1}$. However, since $F$ and $\Phi_{1}$ are functions of time only, it is sufficient to determine $\int_{0}^{1} c_{\mathrm{a}, 1} \mathrm{~d} x$ and $\int_{1}^{\Lambda} c_{\mathrm{c}, 1} \mathrm{~d} x$ instead. Combining the integral of (3.8e) and (3.8f) with (3.8g) leads to the integral conditions:

$$
\frac{\left|\hat{\Omega}_{\text {per }}\right|}{\left|\hat{\Omega}_{\text {per }}\right|+\left|\hat{V}_{\text {per }}\right|} \frac{\mathrm{d}}{\mathrm{d} t} \int_{0}^{1} c_{\mathrm{a}} \mathrm{d} x=-I, \quad \frac{\left|\hat{\Omega}_{\text {per }}\right|}{\left|\hat{\Omega}_{\text {per }}\right|+\left|\hat{V}_{\text {per }}\right|} \frac{\mathrm{d}}{\mathrm{d} t} \int_{1}^{\Lambda} c_{\mathrm{c}} \mathrm{d} x=I \text {. }
$$

Expanding these two conditions to first order and applying the corresponding initial conditions, arising from (3.8h), gives

$$
\begin{aligned}
& \frac{\mathrm{d}}{\mathrm{d} t}\left(\int_{0}^{1} c_{\mathrm{a}, 1} \mathrm{~d} x\right)=0 \\
& \frac{\mathrm{d}}{\mathrm{d} t}\left(\int_{0}^{1} c_{\mathrm{c}, 1} \mathrm{~d} x\right)=\left.0 \int_{1}^{\Lambda} c_{\mathrm{c}, 1} \mathrm{~d} x\right|_{t=0}=0,
\end{aligned}
$$

The above two problems are trivially solved to give

$$
\int_{0}^{1} c_{\mathrm{a}, 1} \mathrm{~d} x=0 \text { and } \int_{1}^{\Lambda} c_{\mathrm{c}, 1} \mathrm{~d} x=0
$$

Rearranging (C.4) to give expressions for $c_{\mathrm{a}, 1}$ and $c_{\mathrm{c}, 1}$ substituting for $\phi_{1}$ from (C.3) and then substituting the result into (C.6) while recalling that $c_{\mathrm{a}, 0}$ and $c_{\mathrm{c}, 0}$ are functions of time only gives rise to the following expressions for $F$ and $\Phi_{1}$ :

$$
F(t)=-\int_{0}^{1} \log c_{0}(x, t)+2 \log \mathcal{G}(x, t) \mathrm{d} x, \quad \Phi_{1}=F(t)+\frac{1}{\Lambda-1} \int_{1}^{\Lambda} \log c_{0}(x, t)+2 \log \mathcal{H}(x, t) \mathrm{d} x,
$$

where $\mathcal{G}$ and $\mathcal{H}$ are given in (C.5). 


\section{Appendix D: Region of small ion concentration}

In order to complete the asymptotic analysis, we investigate in more detail the region $\mu<x<\Lambda$ in which the ion concentration is exponentially small. Here, the asymptotic expansion of the solution takes the form:

$$
\left.\begin{array}{l}
c=\exp \left(-\lambda \zeta_{0}(x, t)-\zeta_{1}(x, t)+\ldots\right) \quad \phi=\phi_{0}(x, t)+\frac{1}{\lambda} \phi_{1}(x, t)+\ldots \quad \Phi=\Phi_{0}+\ldots \\
c_{\mathrm{c}}=c_{\mathrm{c}, 0}+\frac{1}{\lambda} c_{\mathrm{c}, 1}+\ldots \quad G_{\mathrm{c}}=G_{\mathrm{c} 0}+\ldots \quad \tilde{G}_{\mathrm{c}}=\tilde{G}_{\mathrm{c} 0}+\ldots
\end{array}\right\} \quad \text { in } \quad \mu<x<\Lambda,
$$

and to ensure that $c$ is exponentially small we require $\zeta_{0}>0$. Substituting the above into (3.8a) and (3.8f) leads to the results that, to leading order,

$\tilde{G}_{\mathrm{c} 0}=0$ and $\frac{\partial c_{\mathrm{a}}}{\partial t}=0$ in $\mu<x<\Lambda$.

indicating there is no significant charge transfer $\mu<x<\Lambda$.

We note that we can rewrite (3.8a) and (3.8b) in the form (3.8b) and (C.1). Substitution of the expansion (D.1) into (C.1) gives the leading order equation

$\frac{\partial \zeta_{0}}{\partial x}\left(\frac{\partial \phi_{0}}{\partial x}+\frac{\partial \zeta_{0}}{\partial x}\right)=0$

while substitution of (D.1) into (3.8b) implies that $\tilde{G}_{\mathrm{c}}=O\left(\lambda^{2} \mathrm{e}^{-\lambda \zeta_{0}}\right)$. It follows, from (3.8f), that

$\frac{\partial c_{\mathrm{c}, 0}}{\partial t}=0$ in $\mu<x<\Lambda$.

In order to find a closed system of equations for $\zeta_{0}$ and $\phi_{0}$, we require one more relation between these variables in addition to (D.2). This can be found by invoking the Butler-Volmer equation (3.8d) where substitution of the expansion (D.1), combined with the fact that $\tilde{G}_{\mathrm{c}}=O\left(\lambda^{2} \mathrm{e}^{-\lambda \zeta_{0}}\right)$, leads to the balance:

$$
O\left(\lambda^{2} \mathrm{e}^{-\lambda \zeta_{0}}\right)=O\left(\exp \left(-\lambda\left(Z_{0}+\frac{\zeta_{0}}{2}\right)\right)\right)+O\left(\exp \left(-\lambda\left(\frac{\zeta_{0}}{2}-Z_{0}\right)\right)\right)
$$

where $Z_{0}=\frac{1}{2}\left(\phi_{0}-\Phi_{0}+V\left(c_{\mathrm{s}, 0}\right)\right)$.

Given that $\zeta_{0} \geq 0$, the only possible balance occurs between the second and third terms with the result that $Z_{0}=0$. As a consequence we deduce that

$\phi_{0}=\Phi_{0}-V\left(c_{\mathrm{c}, 0}\right)$ and $\frac{\partial \zeta_{0}}{\partial x}=\frac{\partial}{\partial x} V\left(c_{\mathrm{c}, 0}\right)$.

Here, the second equation is a result of combining the first with (D.2). Indeed, we can integrate (D.4) and in order that $c$ matches to the solution in $1<x<\mu$, which is $O(1)$, we require $\left.\zeta_{0}\right|_{x=\mu}=0$. This gives

$\zeta_{0}(x, t)=V\left(c_{\mathrm{c}, 0}(x)\right)-V\left(c_{\mathrm{c}, 0}(\mu, t)\right)$ for $\mu<x<\Lambda$.

Hence, we have shown that the assumption of a free boundary at $x=\mu$ can be systematically justified.

\section{References}

1. Newman JS (2004) Electrochemical systems, 3rd edn. Prentice Hall, New Jersey

2. Botte GG, Subramanian VR, White RE (2000) Mathematical modeling of secondary lithium batteries. Electrochim Acta 45:25952609

3. Dargaville S, Farrell TW (2010) Predicting active material utilization in $\mathrm{LiFePO}_{4}$ electrodes using a multiscale mathematical model. J Electrochem Soc 157:A830-A840

4. Doyle M, Newman J (1996) Analysis of capacity-rate for lithium batteries using simplified models of the discharge process. J Appl Electrochem 27:846-856 
5. Doyle M, Newman J (1996) Comparison of modeling predictions with experimental data from plastic lithium ion cells. J Electrochem Soc 143:1890-1903

6. Farell TW, Please CP (2005) Primary alkaline battery cathodes a simplified model for porous manganese oxide particle discharge. J Electrochem Soc 152:A1930-A1941

7. Fuller TF, Doyle M, Newman J (1994) Simulation and optimisation of the dual lithium ion insertion cell. J Electrochem Soc 141:1-10

8. Fuller TF, Doyle M, Newman J (1994) Relaxation phenomena in lithium-ion-insertion cells. J Electrochem Soc 141:982-990

9. Johansen JF, Farrell TW, Please CP (2006) Modelling of primary alkaline battery cathodes: a simplified model. J Power Sources 156:645-654

10. Pop V, Bergveld HJ, Danilov D, Notten PHL (2008) Battery management systems: accurate state-of-charge indication for batterypowered applications (Philips research book series). Springer, Berlin

11. Srinivasan V, Wang CY (2003) Analysis of electrochemical and thermal behaviour of Li-ion cells. J Electrochem Soc 150:A98A106

12. Owen JR (1997) Rechargeable lithium batteries. Chem Soc Rev 26:259-267

13. Levi MD, Salitra G, Markovsky B, Teller H, Aurbach D, Heider U, Heider L (1999) Solid-state elecrochemical kinetics of Li-ion intercalation into $\mathrm{Li}_{1-x} \mathrm{CoO}_{2}$ : simultaneous application of electroanalytical techniques SSCV, PITT and EIS. J Electrochem Soc 146:1279-1289

14. Kobayashi S, Uchimoto Y (2005) Lithium ion phase-transfer reaction at the interface between the lithium manganese oxide electrode and the nonaqueous electrolyte. J Phys Chem 109:13322-13326

15. Whittingham MS (2004) Lithium battery cathode materials. Chem Rev 104:4271-4301

16. Kang B, Meng YS, Berger J, Grey CP, Ceder G (2006) Electrodes with high power and high capacity for rechargeable lithium batteries. Science 311:977-980

17. Padhi AK, Nanjundaswamy K, Masquelier C, Goodenough JB (1997) Mapping of transition metal redox energies in phosphates with NASICON structure by lithium intercalation. J Electrochem Soc 144:2581-2586

18. Islam MS, Driscoll DJ, Fisher CAJ, Slater PR (2005) Atomic-scale investigation of defects, dopants, and lithium transport in the $\mathrm{LiFePO}_{4}$ olivine-type battery material. Chem Mater 17:5085-5092

19. Singh G, Ceder G, Bazant MZ (2008) Intercalation dynamics in rechargeable battery materials: general theory and phase-transformation waves in $\mathrm{LiFePO}_{4}$. Electrochim Acta 53:7599-7613

20. Morales J, Trcoli R, Franger S, Santos-Pea J (2010) Cycling-induced stress in lithium ion negative electrodes: $\mathrm{LiAl}_{\mathrm{LLiFePO}}$ and $\mathrm{Li}_{4} \mathrm{Ti}_{5} \mathrm{O}_{12} / \mathrm{LiFePO}_{4}$ cells. J Electrochem Acta 9:3075-3082

21. Notten PHL, Roozeboom F, Regtien PPL, Niessen RAH, Baggetto L (2007) 3-D integrated all-solid-state rechargeable batteries. Adv Mater 19:4564-4567

22. Taberna PL, Mitra S, Poizot P, Simon P, Tarascon J-M (2006) High rate capabilities $\mathrm{Fe}_{3} \mathrm{O}_{4}$-based Cu nano-architectured electrodes for lithium-ion battery applications. Nat Mater 5:567-573

23. Whitehead AH, Elliott JM, Owen JR (1999) Nanostructured tin for use as a negative electrode material in Li-ion batteries. J Power Sources 81(82):33-38

24. Chen J-S, Cheh HY (1993) Modelling of cylindrical alkaline cells: IV Dissolution-precipitation model for the anode. J Appl Electrochem 140:1213-1218

25. Johns PA, Roberts MR, Wakizaka Y, Sanders JH, Owen JR (2010) How the electrolyte limits fast discharge in nanostructured batteries and supercapacitors. Electrochem Commun 11:2089-2092

26. Richardson G, King JR (2007) Time-dependent modelling and asymptotic analysis of electrochemical cells. J Eng Math 59:239-275

27. Srinivasan V, Newman J (2004) Discharge model for the lithium iron-phosphate electrode. J Electrochem Soc 151:A1517-A1529

28. Galinski M, Lewandowski A, Stepniak I (2006) Ionic liquids as electrolytes. Electrochim Acta 51:5567-5580

29. Bang HJ, Donepudi VS, Prakash J (2002) Preparation and characterization of partially substituted $\mathrm{Li} \mathrm{M}_{y} \mathrm{Mn}_{2-y} \mathrm{O}_{4}(\mathrm{M}=\mathrm{Ni}, \mathrm{Co}$, Fe) spinel cathodes for Li-ion batteries. Electrochim Acta 48:443-451

30. Lee SH, Cheong HM, Tracy CE, Maschernhas A, Pitts JR, Jorgensen G, Deb SK (2000) Alternating current impedence and Raman spectroscopic study on electrochromic a-WO 3 films. Appl Phys Lett 76:3908-3910

31. Barker J, Pynenburg R, Koksbang R, Saidi MY (1996) An electrochemical investigation into the lithium insertion properties of $\mathrm{Li}_{x} \mathrm{CoO}_{2}$. Electrochim Acta 41:2481-2488

32. Levi MD, Aurbach D (1997) Diffusion coefficients of lithium ions during intercalation into graphite derived from the simultaneous measurements and modeling of electrochemical impedance and potentiostatic intermittent titration of thin graphite electrodes. $\mathrm{J}$ Phys Chem 101:4641-4647

33. Cole JD (1995) Limit process expansions and homogenization. SIAM J Appl Maths 55:410-424

34. Ptashnyk M, Roose T (2010) Derivation of macroscopic model for transport of strongly sorbed solutes in the soil using homogenization techniques. SIAM J Appl Math 70:2097-2118

35. Ptashnyk M, Roose T, Kirk GJD (2010) Diffusion of strongly sorbed solutes in soil: a dual porosity model allowing for slow access to sorption sites and time-dependent sorption reactions. Eur J Soil Sci 61:108-119

36. Wang CY, Gu WB, Liaw BY (1998) Micro-macroscopic coupled modeling of batteries and fuel cells. J Electrochem Soc 145:34073417

37. Atkins P, de Paula J (2001) Atkins' physical chemistry, 7th edn. OUP, New York 